\title{
Optimal control of trading algorithms: a general impulse control approach
}

\author{
Bruno Bouchard* Ngoc-Minh Dang ${ }^{\dagger}$ and Charles-Albert Lehalle ${ }^{\ddagger}$
}

November 14, 2009

\begin{abstract}
We propose a general framework for intra-day trading based on the control of trading algorithms. Given a generic parameterized algorithm, we control the dates $\left(\tau_{i}\right)_{i}$ at which it is launched, the length $\left(\delta_{i}\right)_{i}$ of the trading period and the value of the parameters $\left(\mathcal{E}_{i}\right)_{i}$ kept during the time interval $\left[\tau_{i}, \tau_{i}+\delta_{i}[\right.$. This gives rise to a non-classical impulse control problem where not only the regime $\mathcal{E}_{i}$ but also the period $\left[\tau_{i}, \tau_{i}+\delta_{i}[\right.$ has to be determined by the controller at the impulse time $\tau_{i}$. We adapt the weak dynamic programming principle of Bouchard and Touzi (2009) to our context and provide a characterization of the associated value function as a discontinuous viscosity solution of a system of PDEs with appropriate boundary conditions, for which we prove a comparison principle. We also propose a numerical scheme for the resolution of the above system and show that it is convergent. We finally provide an example of application to a problem of optimal stock trading with a non-linear market impact function.
\end{abstract}

Key words: optimal impulse control, discontinuous viscosity solutions, intra-day trading.

Mathematical subject classifications: 93E20, 49L25, 91B28.

\section{Introduction}

The financial crisis put the emphasis on the role of market liquidity during the exchanges of financial products. Since the formalisation of the so-called optimal liquidation problem in the late nineties (see [4] and [6]), each inclusion of a new effect in the "optimal-control-oriented" original framework gave birth to a specific extension. It has been the case for taking into account some statistical effects [15], specific properties of the price diffusion process as being Ornstein Uhlenbeck [14], information at an orderbook level [11], or Bayesian estimation of the market trend [3].

This paper presents a generic framework that has the ambition to be able to include any effect.

In short, the optimal liquidation problem arises when one needs to buy and sell an arbitrary combination of stocks on a finite time horizon. The structure of auction markets implies the existence of a "liquidity effect" often called "market impact" or "execution costs" [17]: buying an amount $v$ of shares of a stock during an interval of length $\delta$ beginning at time $\tau$ generates

* CEREMADE, Université Paris Dauphine and CREST-ENSAE, bouchard@ceremade.dauphine.fr

${ }^{\dagger}$ CEREMADE, Université Paris Dauphine and CA Cheuvreux, dang@ceremade.dauphine.fr

${ }^{\ddagger} \mathrm{CA}$ Cheuvreux, clehalle@cheuvreux.com 
an over-cost that is a function of the "market depth" over $[\tau, \tau+\delta]$. Some empirical studies have shown that the combination of the volatility $\sigma$, the bid-ask spread $\psi_{B A}$ and the usual traded volume $V$ over this time interval are an efficient proxy of the market depth [12], giving birth to the following class of model linking the market impact $\eta(v)$ to market-observations:

$$
\eta(v ; \tau, \tau+\delta)=\alpha \cdot \psi_{B A}(\tau, \tau+\delta)+\kappa \cdot \sigma(\tau, \tau+\delta)\left(\frac{v}{V(\tau, \tau+\delta)}\right)^{\gamma}
$$

where $\alpha, \kappa$ and $\gamma$ are positive parameters taking different values from one stock to another (see [10] to have an overview of several models and for the viability domains of their parameters). This market impact has to be added to the last traded price $S_{\tau}$ to obtain the average price to buy the needed $v$ shares.

One may want to trade slowly to avoid paying too much of this premium to other market participants (increasing $\delta$ decreases the participation rate $\rho(\tau)=v / V(\tau, \tau+\delta)$ ), but it will generate a more important exposure to market moves.

The considered utility function to optimize (rendering the balance between market impact that demands to trade slowly and market risk that demands to trade rapidly) and the hypothesis on the price formation process (dependent moves of the price, the volatility, the bid-ask spread and the traded volumes) generate sophisticated stochastic optimization problems that are the core of the quantitative trading field including the optimal portfolio liquidation problem.

The original framework is built on an a priori discretization of the trading phases in $N$ time intervals as the inclusion of some specific effects like taking into account real time analytics came from continuous time models [1].

None of those two approaches is optimal. Firstly because the root of the market impact is the market microstructure that takes place at an event-driven and consequently discretized time scale [18]. Also because an a priori discretization is not compatible with the algorithmic trading processes, that are based on the launch of "slices" of buy or sell orders in the markets, whose durations are not the same over the whole trading phase.

From the point of view of this paper, the algorithmic trading process is the one of switching between those states:

- the passive regime: where no slice is in the market. During such a state, the price formation process will continue to take place without interaction with the controlled order.

- the active regime: with a parametrized slice in the market. The duration of each slice is bounded from below by a constant $\underline{\delta}$, and the characteristics of a slice are chosen just before its launch and cannot be modified until it ends.

The framework proposed in this paper takes precisely into account the reality of the "slicing" of parent orders into child orders and their interactions with the market microstructure. It also offers the capability to model the market underlying moves via time-continuous models. The generic market model $X^{\nu}$ used in this paper is the one of the strong solution of a controlled stochastic differential equation with jumps:

$$
X^{\nu}(t)=x_{0}+\int_{0}^{t}\left(b\left(X^{\nu}(s), \nu_{s}\right) d s+a\left(X^{\nu}(s), \nu_{s}\right) d W_{s}\right)+\sum_{i} \beta\left(X^{\nu}\left(\tau_{i}^{\nu}-\right), \mathcal{E}_{i}^{\nu}, \delta_{i}^{\nu}\right) \mathbf{1}_{\tau_{i}^{\nu} \leq t}
$$

The control $\nu$, which corresponds to the current regime, is identified to sequence of triplets $\left(\tau^{\nu}, \mathcal{E}^{\nu}, \delta^{\nu}\right)$ where $\tau^{\nu}$ is the launch time of a slice, $\delta^{\nu}$ the duration of the slice, and $\mathcal{E}^{\nu}$ the value of the parameters of the slice (e.g. its participation rate). 
The aim of the controller is to maximize the expected value of a gain functional of the form

$$
g\left(X^{\nu}(T)\right)+\sum_{\tau_{i}^{\nu}+\delta_{i}^{\nu} \leq T} f\left(X^{\nu}\left(\tau_{i}^{\nu}+\delta_{i}^{\nu}\right), \mathcal{E}_{i}^{\nu}\right) .
$$

This model can be used to control the launch of any type of slices, from very simple ones (as in [4]) to the launch of "trading robots" (incorporating Smart Order Routing capabilities like the ones described in [2] and [16]). Using such algorithmic trading models for the launch of simple slices will lead to an optimized not-uniformly sampled sequence of slices, taking into account the market rhythm.

Such a model can also be used to control "meta trading algorithms", that are dedicated to the optimal launch of sequences of traditional algorithms. This paper is the first to proposed a framework to optimize such meta-algo, despite the fact that a lot of trading desk demand such optimal combinations of well-known algorithms.

On the other hand, the continuous-time aspect of the market model opens the door to the use of traditional models and tools from quantitative finance.

From the mathematical point of view, it gives rise to a non-classical impulse control problem. When the current regime is the passive one, i.e. the trading algorithm is not running, the controller can launch it at any moment $\tau_{i}$ with a given set of parameters $\mathcal{E}_{i}$ and for a period of length $\delta_{i}$. This leads to a characterization of the value function in terms of a standard quasi-variational inequality in the region corresponding to the passive regime. However, once the algorithm is launched, no change in the value of the parameters can be made before the end of the period $\left[\tau_{i}, \tau_{i}+\delta_{i}[\right.$. This implies that the value function satisfies a linear parabolic equation on the active region.

In this paper, we provide a rigorous characterization of the value function as a discontinuous viscosity solution of such equations, together with suitable boundary conditions. To this end, we adapt the approach of [7] who proposes a weak version of the dynamic programming principle. The main advantage of this weak formulation is that it does not require any apriori continuity of the value function. We also provide a comparison principle for the above equations and construct a finite difference numerical scheme, which we prove to be convergent. The rest of the paper is organized as follows. The model is described in Section 2. In Section 3, we provide the PDE characterization of the value function and the associated comparison principle. The proofs of these results are given in Section 4. The numerical scheme is studied in Section 5 . In the last section, we discuss an example of application to a particular model of optimal stock liquidation. It shows how the proposed framework naturally allows a real-time adaptive control of the trading algorithm, by switching optimally after the end of each slice given the current state of the market.

Notations: All over this paper, we shall use the following notations. Given $x \in \mathbb{R}^{k}$, for $k$ given by the context, we denote by $|x|$ its Euclidean norm and by $B_{r}(x)$ the open ball of center $x$ and radius $r>0$. The scalar product is denoted by $\langle\cdot, \cdot\rangle$. Given a set $A \subset \mathbb{R}^{k}$, we denote by $\partial A$ its boundary. Given $d \in \mathbb{N}$, we denote by $\mathbb{M}^{d}$ the set of $d$-dimensional square matrices. For $M \in \mathbb{M}^{d}, M^{*}$ is the associated transposed matrix. For a function $(t, x, y) \in \mathbb{R}_{+} \times \mathbb{R}^{d} \times \mathbb{R}^{k} \mapsto \varphi(t, x, y)$, we denote by $D \varphi$ and $D^{2} \varphi$ its gradient and Hessian matrix with respect to $x$, whenever they are well defined. The other partial derivatives will be written by using standard notations. 


\section{Problem formulation}

Let $(\Omega, \mathcal{F}, \mathbb{P})$ be a probability space supporting a $d$-dimensional Brownian motion $W, d \geq 1$. Let $\mathbb{F}:=\left(\mathcal{F}_{t}\right)_{t \geq 0}$ denote the right-continuous complete filtration generated by $W$, and let $T>0$ be a finite time horizon.

\subsection{Control policies}

A control policy of the trading algorithm is described by a non-decreasing sequence of stopping times $\left(\tau_{i}\right)_{i \geq 1}$ and a sequence of $E \times[\underline{\delta}, \infty)$-valued random variables $\left(\mathcal{E}_{i}, \delta_{i}\right)_{i \geq 1}$. The stopping times $\tau_{i}$ describe the times at which an order is given to the algorithm, $\mathcal{E}_{i}$ is the value of the parameters with which the algorithm is run and $\delta_{i}$ the length of the period (latency period) during which it is run with the value $\mathcal{E}_{i}$. The set $E$ is a compact subset of $\mathbb{R}^{d}, d \geq 1$, which represents the possible values of the parameters, the quantity

$$
0<\underline{\delta}<T
$$

denotes the minimum length of the time period during which the algorithm can be run. To be consistent we impose that

$$
\tau_{i}+\delta_{i} \leq \tau_{i+1} \text { and }\left(\delta_{i}>0 \Rightarrow \tau_{i}+\delta_{i} \leq T\right), i \geq 1 .
$$

The first condition expresses the fact that a new order can not be given before the end of the time period associated to the previous order. The second one means that an order should be given only if it ends before the final time horizon $T$. As usual the value of the parameters and the size of the latency period can not be chosen in some anticipative way, i.e. we impose that

$$
\left(\delta_{i}, \mathcal{E}_{i}\right) \text { is } \mathcal{F}_{\tau_{i}} \text {-measurable, } i \geq 1 \text {. }
$$

At time $t \in\left[\tau_{i}, \tau_{i}+\delta_{i}\right)$, the value of the parameter of the trading algorithm is denoted by $\nu_{t}$. For $t \in A\left(\left(\tau_{i}, \delta_{i}\right)_{i \geq 1}\right)$, defined as

$$
A\left(\left(\tau_{i}, \delta_{i}\right)_{i \geq 1}\right):=\mathbb{R}_{+} \backslash\left(\bigcup_{i \geq 1}\left[\tau_{i}, \tau_{i}+\delta_{i}\right)\right),
$$

we set $\nu_{t}=\varpi$, where $\varpi \in \mathbb{R}^{d} \backslash E$ can be viewed as a cimetary point, recall that $E$ is compact. It follows that the value of the parameters of the trading algorithm $\nu$ can be written as

$$
\nu_{t}=\varpi \mathbf{1}_{t \in A\left(\left(\tau_{i}, \delta_{i}\right)_{i \geq 1}\right)}+\sum_{i \geq 1} \mathbf{1}_{t \in\left[\tau_{i}, \tau_{i}+\delta_{i}\right)} \mathcal{E}_{i}, t \in[0, T],
$$

where $\nu_{t}=\varpi$ means that the algorithm is not running at time $t$.

We denote by $\mathcal{S}$ the set of adapted processes $\nu$ that can be written in the form (2.3) for some sequence of stopping times $\left(\tau_{i}\right)_{i \geq 1}$ and of $E \times[\underline{\delta}, \infty)$-valued random variables $\left(\delta_{i}, \mathcal{E}_{i}\right)_{i \geq 1}$ satisfying (2.1) and (2.2).

For ease of notations, we shall now write

$$
\left(\tau_{i}^{\nu}, \delta_{i}^{\nu}, \mathcal{E}_{i}^{\nu}\right)_{i \geq 1} \text { the sequence associated to } \nu \in \mathcal{S},
$$


and define, for all stopping times $\vartheta_{1}$ and $\vartheta_{2}$ satisfying $\vartheta_{1} \leq \vartheta_{2} \mathbb{P}$-a.s., the quantity

$$
\mathbb{I}_{\vartheta_{1}, \vartheta_{2}}^{\nu}:=\left\{i \geq 1: \vartheta_{1}<\tau_{i}^{\nu}+\delta_{i}^{\nu} \leq \vartheta_{2}\right\},
$$

which denotes the number of orders whose execution ends between $\vartheta_{1}$ and $\vartheta_{2}$.

Remark 2.1. Note that the constraint $\delta_{i}^{\nu} \geq \underline{\delta}$ for all $i \geq 1$ and $\nu \in \mathcal{S}$ implies that

$$
\left|\mathbb{I}_{0, T}^{\nu}\right| \leq\left|\left\{\tau_{i}^{\nu} \leq T, i \geq 1\right\}\right| \leq T / \underline{\delta} .
$$

For ease of notations, we also set

$$
\bar{E}:=E \cup\{\varpi\},
$$

and introduce the processes

$$
\Delta_{t}^{\nu}:=\sum_{i \geq 1}\left[\tau_{i}^{\nu}+\delta_{i}^{\nu}-t\right]^{+} \mathbf{1}_{t \geq \tau_{i}^{\nu}}, t \in[0, T] .
$$

The quantity $\Delta_{t}^{\nu}$ denotes the remaining latency period during which no new order can be passed to the algorithm. When $\Delta_{t}^{\nu}>0$, the algorithm is running with a value of the parameters $\nu_{t}$. When $\Delta_{t}^{\nu}=0$, the algorithm is not running anymore and a new order can be passed.

The following picture sums up the dynamics of the control.

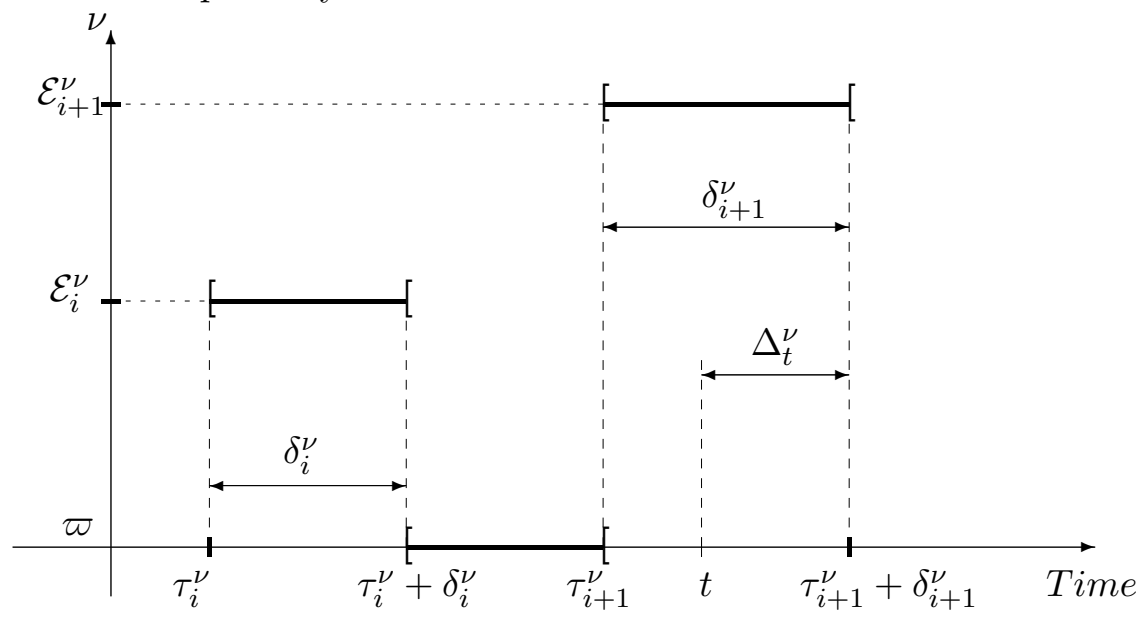

\subsection{Output of the trading algorithm and gain function}

Given some initial data $(t, x) \in[0, T] \times \mathbb{R}^{d}$, the output of the trading algorithm associated to some control policy $\nu \in \mathcal{S}$ is defined as the strong solution $X_{t, x}^{\nu}$ on $[0, T]$ of the stochastic differential equation

$$
X_{t, x}^{\nu}(s)=x+\mathbf{1}_{s \geq t}\left(\int_{t}^{s} b\left(X_{t, x}^{\nu}(r), \nu_{r}\right) d r+\int_{t}^{s} a\left(X_{t, x}^{\nu}(r), \nu_{r}\right) d W_{r}+\sum_{i \geq 1} \beta\left(X_{t, x}^{\nu}\left(\tau_{i}^{\nu}-\right), \mathcal{E}_{i}^{\nu}, \delta_{i}^{\nu}\right) \mathbf{1}_{t<\tau_{i}^{\nu} \leq s}\right),
$$


where $b, \beta: \mathbb{R}^{d} \times \bar{E} \times[\underline{\delta}, T] \mapsto \mathbb{R}^{d}$ and $a: \mathbb{M}^{d} \times \bar{E} \mapsto \mathbb{M}^{d}$ are continuous functions satisfying, for all $x, x^{\prime} \in \mathbb{R}^{d}, e, e^{\prime} \in \bar{E}, \delta, \delta^{\prime} \in[\underline{\delta}, T]$,

$(2.5)\left\{\begin{aligned}\left.\left|\psi(x, e, \delta)-\psi\left(x^{\prime}, e, \delta\right)\right|\right) & \leq K\left|x-x^{\prime}\right| \\ |\psi(x, e, \delta)| & \leq K(1+|x|) \\ \left|\psi(x, e, \delta)-\psi\left(x, e^{\prime}, \delta^{\prime}\right)\right| & \leq K(1+|x|)\left(\left|e-e^{\prime}\right|+\left|\delta-\delta^{\prime}\right|\right)\end{aligned} \quad\right.$ for $\psi=b, a, \beta$,

for some $K>0$.

Remark 2.2. Observe that $X^{\nu}$ does not jump when the regime is switched to the passive regime $\varpi$.

We do not differentiate here between the components that correspond to real outputs of the algorithm (cumulated gains, cumulated volumes executed by the algorithm, etc...) and others that simply describe the evolution of financial data or market factors (prices of the traded assets, global traded volumes on the markets, volatilities, etc...).

The jumps on the dynamics are introduced to model the change in the initial conditions on the variable of interest for the trading algorithm when it is launched (e.g. volume to be executed between $\tau_{i}^{\nu}$ and $\tau_{i}^{\nu}+\delta_{i}^{\nu}$ ).

Remark 2.3. Note that (2.5), the fact that $E$ is bounded and Remark 2.1 imply that, for all $t \in[0, T], x \in \mathbb{R}^{d}$ and $\nu \in \mathcal{S}$,

$$
\mathbb{E}\left[\sup _{s \in[t, T]}\left|X_{t, x}^{\nu}(s)\right|^{p}\right] \leq C_{K}^{p}\left(1+|x|^{p}\right)
$$

where $C_{K}^{p}$ depends only on $K$ and $p \geq 1$.

The aim of the controller is to maximize the expected value of the gain functional

$$
\nu \in \mathcal{S} \mapsto \Pi_{t, x}(\nu):=g\left(X_{t, x}^{\nu}(T)\right)+\sum_{i \in \mathbb{I}_{t, T}^{\nu}} f\left(X_{t, x}^{\nu}\left(\tau_{i}^{\nu}+\delta_{i}^{\nu}-\right), \mathcal{E}_{i}^{\nu}\right)
$$

with the usual convention $\sum_{\emptyset}=0$, among the set

$$
\mathcal{S}_{t, \delta, e}:=\left\{\nu \in \mathcal{S}: \nu_{s}=e \text { for } s \in[t, t+\delta) \text { and } \Delta_{t+\delta}^{\nu}=0\right\},
$$

where $(\delta, e) \in \mathbb{R}_{+} \times \bar{E}$ denotes the initial state of the remaining latency time and value of the parameters.

Here, $g$ and $f$ are assumed to be continuous on $\mathbb{R}^{d} \times \bar{E}$ and to satisfy, for some $\gamma>0$,

$$
\sup _{(x, e) \in \mathbb{R}^{d} \times E} \frac{|f(x, e)|+|g(x)|}{1+|x|^{\gamma}}<\infty, f(\cdot, \varpi)=0 .
$$

In view of (2.6), this ensures that the quantity

$$
J(t, x ; \nu):=\mathbb{E}\left[\Pi_{t, x}(\nu)\right]
$$

is well defined for all $\nu \in \mathcal{S}$ and satisfies

$$
|J(t, x ; \nu)| \leq C_{K}^{\gamma}\left(1+|x|^{\gamma}\right)
$$


where $C_{K}^{\gamma}$ depends only on $K$ and $\gamma$.

For technical reason related to the dynamic programming principle, see [7] and the proof of Lemma 4.1 below, we shall restrict to admissible trading strategies $\nu \in \mathcal{S}_{t, \delta, e}$ such that $\nu$ is independent on $\mathcal{F}_{t}$, see Remark 5.2 in [7]. We denote by $\mathcal{S}_{t, \delta, e}^{a}$ the associated set of controls and therefore define the value function as:

$$
V(t, x, \delta, e):=\sup _{\nu \in \mathcal{S}_{t, \delta, e}^{a}} \mathbb{E}\left[\Pi_{t, x}(\nu)\right]
$$

We refer to Section 6 for examples of application.

Remark 2.4. It follows from (2.8) that there exists $C_{K}^{\gamma}>0$ which depends only on $K$ and $\gamma$ such that

$$
|V(t, x, \delta, e)| \leq C_{K}^{\gamma}\left(1+|x|^{\gamma}\right) \quad \text { for all }(t, x, \delta, e) \in[0, T] \times \mathbb{R}^{d} \times \mathbb{R}_{+} \times \bar{E} \text { s.t. } \mathcal{S}_{t, \delta, e}^{a} \neq \emptyset .
$$

Note that for $\delta=T-t$ and $e \in E,(2.1)$ implies that

$$
V(t, x, T-t, e)=\mathcal{V}(t, x, e):=\mathbb{E}\left[g\left(\mathcal{X}_{t, x}^{e}(T)\right)+f\left(\mathcal{X}_{t, x}^{e}(T), e\right)\right],
$$

where $\mathcal{X}_{t, x}^{e}$ is the solution of

$$
\mathcal{X}_{t, x}^{e}(s)=x+\int_{t}^{s} b\left(\mathcal{X}_{t, x}^{e}(r), e\right) d r+\int_{t}^{s} a\left(\mathcal{X}_{t, x}^{e}(r), e\right) d W_{r}, s \in[t, T] .
$$

Remark 2.5. Under (2.5), the continuity assumption on $f, g$ and (2.7), it follows from standard arguments that the auxiliary value function $\mathcal{V}$ is continuous, and that, for each $e \in E$, it is a viscosity solution of

$$
-\mathcal{L}^{e} \varphi(t, x)=0 \text { on }[0, T) \times \mathbb{R}^{d}, \varphi(T, x)=g(x)+f(x, e) \text { on } \mathbb{R}^{d},
$$

where, for $e \in \bar{E}$, and a smooth function $\varphi$,

$$
\mathcal{L}^{e} \varphi(t, x):=\frac{\partial}{\partial t} \varphi(t, x)+\langle b(x, e), D \varphi(t, x)\rangle+\frac{1}{2} \operatorname{Tr}\left[a a^{*}(x, e) D^{2} \varphi(t, x)\right] .
$$

\section{Viscosity characterization of the value function}

The aim of this section, is to provide a PDE characterization of the value function $V$. Before to state our main result, we need to introduce some additional notations and definitions.

In view of (2.1), (2.9) and the constraint that the latency period should be bigger than $\underline{\delta}$, the natural domain of definition of the value function $V$ is

$$
D:=\left\{(t, x, \delta, e) \in[0, T) \times \mathbb{R}^{d} \times(((0, \infty) \times E) \cup\{0, \varpi\}): \underline{\delta} \leq t+\delta<T \text { or } e=\varpi\right\},
$$

which can be decomposed in two main regions. We call the active region, the region where $\delta>0$ and $e \neq \varpi$ :

$$
D_{E,>0}:=\left\{(t, x, \delta, e) \in[0, T) \times \mathbb{R}^{d} \times(0, \infty) \times E: \underline{\delta} \leq t+\delta<T\right\} .
$$


It corresponds to the set of initial conditions where the algorithm is running and the controller has to wait the end of the latency period before passing a new order. We call the passive region, the region where $e=\varpi$, and therefore $\delta=0$ :

$$
D_{\varpi}:=[0, T) \times \mathbb{R}^{d} \times\{0, \varpi\} .
$$

It corresponds to the set of initial conditions where the algorithm is not running and can be launched immediately with a new set of parameters. These two regions are complemented by the natural boundaries of the active region when $\delta \rightarrow 0$ and $t+\delta \rightarrow T$ :

$D_{E, 0}:=[\underline{\delta}, T) \times \mathbb{R}^{d} \times\{0\} \times E, D_{E, T}:=\left\{(t, x, \delta, e) \in[0, T) \times \mathbb{R}^{d} \times(0, \infty) \times E: \underline{\delta} \leq t+\delta=T\right\}$, and the time boundary:

$$
D_{T}:=\{T\} \times \mathbb{R}^{d} \times \mathbb{R}_{+} \times \bar{E} .
$$

The closure of the natural domain of definition of the value function $V$ is therefore

$$
\bar{D}:=\left\{(t, x, \delta, e) \in[0, T] \times \mathbb{R}^{d} \times \mathbb{R}_{+} \times \bar{E}: \underline{\delta} \leq t+\delta \leq T \text { or } e=\varpi\right\} .
$$

As usual, we shall rely on the dynamic programming principle, see Lemma 4.1 below for a precise statement, to deduce the behavior of the value function on each component of $\bar{D}$ :

$$
V(t, x, \delta, e)=\sup _{\nu \in S_{t, \delta, e}^{a}} \mathbb{E}\left[V\left(\vartheta, X_{t, x}^{\nu}(\vartheta), \Delta_{\vartheta}^{\nu}, \nu_{\vartheta}\right)+\sum_{i \in \mathbb{T}_{t, \vartheta}^{\nu}} f\left(X_{t, x}^{\nu}\left(\tau_{i}^{\nu}+\delta_{i}^{\nu}\right), \mathcal{E}_{i}^{\nu}\right)\right]
$$

for any $[t, T]$-valued stopping time $\vartheta$.

In the passive region. For $(t, x, \delta, e) \in D_{\varpi}$, the controller can immediately launch the trading algorithm with a new set of parameters $\left(\delta^{\prime}, e^{\prime}\right) \in[\underline{\delta}, T-t] \times E$. Taking $\vartheta=t$ in (3.1) thus implies that

$$
V(t, x, 0, \varpi) \geq \mathcal{M}[V](t, x)
$$

where

$$
\mathcal{M}[V](t, x):=\sup _{\left(\delta^{\prime}, e^{\prime}\right) \in[\underline{\delta}, T-t] \times E} V\left(t, x+\beta\left(x, e^{\prime}, \delta^{\prime}\right), \delta^{\prime}, e^{\prime}\right),
$$

with the usual convention $\sup \emptyset=-\infty$. The controller can also decide to wait before passing a new order to the algorithm, i.e. choose $\nu=\varpi$ on some time interval $\left[t, t+\delta^{\prime}\right)$ with $\delta^{\prime}>0$. In view of (3.1) applied to an arbitrarily small stopping time $\vartheta<t+\delta^{\prime}$, this implies that

$$
-\mathcal{L}^{\varpi} V(t, x, 0, \varpi) \geq 0
$$

The dynamic programming principle (3.1) formally implies that one of the two above choices should be optimal, i.e.

$$
\min \left\{-\mathcal{L}^{\varpi} V(t, x, 0, \varpi) ; V(t, x, 0, \varpi)-\mathcal{M}[V](t, x)\right\}=0 .
$$

In the active region. For $(t, x, \delta, e) \in D_{E,>0}$, the controller can not change the parameter of the algorithm before the end of the initial latency period $\delta>0$. Choosing $\vartheta$ arbitrarily small in (3.1) thus implies that $V$ should satisfy

$$
\left(-\mathcal{L}^{e}+\frac{\partial}{\partial \delta}\right) V(t, x, \delta, e)=0 .
$$


It is naturally complemented with the boundary conditions

$$
V(t, x, \delta, e)=V(t, x, 0, \varpi)+f(x, e), \text { if }(t, x, \delta, e) \in D_{E, 0},
$$

and

$$
V(t, x, \delta, e)=\mathcal{V}(t, x, e), \quad \text { if }(t, x, \delta, e) \in D_{E, T},
$$

recall (2.9).

Terminal boundary condition. As usual, the boundary condition as $t \uparrow T$ should be given by the terminal condition:

$$
V(t, x, \delta, e)=g(x)+f(x, e), \text { if }(t, x, \delta, e) \in D_{T},
$$

where we recall that $f(\cdot, \varpi)=0$ by convention.

The above discussion shows that $V$ should solve the equation

$$
\mathcal{H} \varphi=0
$$

on $\bar{D}$, where, for a smooth function $\varphi$ defined on $\bar{D}$,

$$
\mathcal{H} \varphi(t, x, \delta, e):=\left\{\begin{array}{ccc}
\left(-\mathcal{L}^{e}+\frac{\partial}{\partial \delta}\right) \varphi(t, x, \delta, e) & \text { on } & D_{E,>0}, \\
\varphi(t, x, \delta, e)-\varphi(t, x, 0, \varpi)-f(x, e) & \text { on } & D_{E, 0}, \\
\varphi(t, x, \delta, e)-\mathcal{V}(t, x, e) & \text { on } & D_{E, T}, \\
\min \left\{-\mathcal{L}^{\varpi} \varphi(t, x, \delta, e) ; \varphi(t, x, \delta, e)-\mathcal{M}[\varphi](t, x)\right\} & \text { on } & D_{\varpi}, \\
\varphi(t, x, \delta, e)-g(x)-f(x, e) & \text { on } & D_{T} .
\end{array}\right.
$$

However, since $V$ may not be smooth, it has to be stated in terms of viscosity solutions, see [8], in the following sense.

Definition 3.1. We say that a lower-semicontinuous (resp. upper-semicontinuous) function $U$ on $\bar{D}$ is a viscosity supersolution (resp. subsolution) of (3.2) on $\bar{D}$ if for any function $\varphi \in C^{1,2,1,0}\left([0, T] \times \mathbb{R}^{d} \times \mathbb{R}_{+} \times \bar{E}\right)$ and $\left(t_{0}, x_{0}, \delta_{0}, e_{0}\right) \in \bar{D}$, which achieves a global minimum (resp. maximum) of $U-\varphi$ on $\bar{D}$ such that $(U-\varphi)\left(t_{0}, x_{0}, \delta_{0}, e_{0}\right)=0$, we have

$$
\mathcal{H} \varphi\left(t_{0}, x_{0}, \delta_{0}, e_{0}\right) \geq 0 \quad\left(\text { resp. } \mathcal{H} \varphi\left(t_{0}, x_{0}, \delta_{0}, e_{0}\right) \leq 0\right) .
$$

If $U$ is continuous, we say that it is a viscosity solution of (3.2) if it is a super- and a subsolution.

In all this paper, we shall say that a function $\varphi$ is smooth if it belongs to $C^{1,2,1,0}\left([0, T] \times \mathbb{R}^{d} \times\right.$ $\left.\mathbb{R}_{+} \times \bar{E}\right)$.

As usual, showing that $V$ is a-priori continuous is a rather difficult task. As a first step, we shall therefore prove the super- and subsolution property only for the upper- and lowersemicontinuous enveloppes $V^{*}$ and $V_{*}$ of $V$ defined as

$$
\begin{aligned}
V^{*}(t, x, \delta, e) & :=\limsup _{\left(t^{\prime}, x^{\prime}, \delta^{\prime}, e^{\prime}\right) \in D \rightarrow(t, x, \delta, e)} V\left(t^{\prime}, x^{\prime}, \delta^{\prime}, e^{\prime}\right) \\
V_{*}(t, x, \delta, e) & :=\liminf _{\left(t^{\prime}, x^{\prime}, \delta^{\prime}, e^{\prime}\right) \in D \rightarrow(t, x, \delta, e)} V\left(t^{\prime}, x^{\prime}, \delta^{\prime}, e^{\prime}\right), \quad(t, x, \delta, e) \in \bar{D} .
\end{aligned}
$$

Theorem 3.2. The function $V_{*}\left(\right.$ resp. $\left.V^{*}\right)$ is a vicosity supersolution (resp. subsolution) of (3.2) on $\bar{D}$. 
The following comparison result combined with Remark 2.4 insures a-posteriori that $V$ is continuous and that it is the unique viscosity solution of (3.2) on $\bar{D}$ with polynomial growth.

Theorem 3.3. Let $u$ and $v$ be respectively a lower semicontinuous viscosity supersolution of (3.2) on $\bar{D}$ and a upper-semicontinuous viscosity subsolution of (3.2) on $\bar{D}$. Assume that $v^{+}$ and $u^{-}$have polynomial growth. Then, $u \geq v$ on $\bar{D}$.

\section{Proof of the viscosity characterization}

\subsection{Dynamic programming}

As usual, the derivation of the partial differential equation relies on the so-called dynamic programming principle, a formal version of which is given in (3.1) above. In this section, we provide a rigorous formulation which follows ideas introduced in [7]. Namely, we only provide a weak formulation in terms of test functions. The main advantage of this approach is that it does not require any regularity on the value function $V$ itself, but only some lowersemicontinuity of the objective function $J(\cdot ; \nu)$, see below. We refer to [7] for a general discussion.

Lemma 4.1 (Weak Dynamic Programming Principle). Fix $(t, x, \delta, e) \in D$ and let $\left\{\vartheta^{\nu}, \nu \in\right.$ $\left.\mathcal{S}_{t, \delta, e}^{a}\right\}$ be a family of $[t, T]$-valued stopping times independent of $\mathcal{F}_{t}$. Then, we have

$$
V(t, x, \delta, e) \leq \sup _{\nu \in \mathcal{S}_{t, \delta, e}^{a}} \mathbb{E}\left[\left[V^{*}, g\right]\left(\vartheta^{\nu}, X_{t, x}^{\nu}\left(\vartheta^{\nu}\right), \Delta_{\vartheta^{\nu}}^{\nu}, \nu_{\vartheta^{\nu}}\right)+\sum_{i \in \mathbb{I}_{t, \vartheta^{\nu}}^{\nu}} f\left(X_{t, x}^{\nu}\left(\tau_{i}^{\nu}+\delta_{i}^{\nu}-\right), \mathcal{E}_{i}^{\nu}\right)\right],
$$

where $\left[V^{*}, g\right](s, \cdot):=V^{*}(s, \cdot) \mathbf{1}_{s<T}+g \mathbf{1}_{s=T}$, and

$(4.2) \sup _{\nu \in \mathcal{S}_{t, \delta, e}^{a}} \mathbb{E}\left[\varphi\left(\vartheta^{\nu}, X_{t, x}^{\nu}\left(\vartheta^{\nu}\right), \Delta_{\vartheta^{\nu}}^{\nu}, \nu_{\vartheta^{\nu}}\right)+\sum_{i \in \mathbb{I}_{t, \vartheta^{\nu}}} f\left(X_{t, x}^{\nu}\left(\tau_{i}^{\nu}+\delta_{i}^{\nu}-\right), \mathcal{E}_{i}^{\nu}\right)\right] \leq V(t, x, \delta, e)$

for all upper semi-continuous function $\varphi$ such that $V \geq \varphi$ on $\bar{D}$.

As in [7], the proof of the above result relies on some lower-semicontinuity property of the function $J$. Because of the latency time $\delta$, we can however not apply their result directly and need to adapt their arguments by exploiting the lower-semicontinuity of the map

$$
(t, x, \delta, e, \nu) \in \bar{D} \times \mathcal{S} \mapsto J\left(t, x, \mathscr{P}_{t+\delta}^{e}(\nu)\right)
$$

where, for $s_{1} \leq s_{2} \in[0, T]$,

$$
\mathscr{P}_{s_{1}, s_{2}}^{e}: \nu \in \mathcal{S} \mapsto \mathscr{P}_{s_{1}, s_{2}}^{e}(\nu):=e \mathbf{1}_{\left[0, s_{1}\right)}+\varpi \mathbf{1}_{\left[s_{1}, s_{2}\right)}+\nu \mathbf{1}_{\left[s_{2}, T\right]} .
$$

Lemma 4.2. Fix $(t, x, \delta, e) \in D$ and $\nu \in \mathcal{S}_{t, \delta, e}$. Let $\left(t_{n}, x_{n}, \delta_{n}, e_{n}\right)_{n \geq 1}$ be a sequence in $D$ such that $\left(t_{n}, x_{n}, \delta_{n}, e_{n}\right) \rightarrow(t, x, \delta, e)$ as $n \rightarrow \infty$ and

$$
t_{n} \leq t \text { and } t_{n}+\delta_{n} \leq t+\delta \text { for all } n \geq 1 .
$$

Then, $\liminf _{n \rightarrow \infty} J\left(t_{n}, x_{n} ; \mathscr{P}_{t_{n}+\delta_{n}, t+\delta}^{e_{n}}(\nu)\right) \geq J(t, x ; \nu)$. 
Proof. We only prove the result in the case where $\delta>0$. The case $\delta=0$ can be handled similarly. In this case we have $t_{n} \leq t<t_{n}+\delta_{n} \leq t+\delta$ for $n$ large enough since $t_{n} \rightarrow t$ and $\delta_{n} \rightarrow \delta>0$. For ease of notations, we set $X:=X_{t, x}^{\nu}$ and $X^{n}:=X_{t_{n}, x_{n}}^{\nu^{n}}$ where $\nu^{n}:=$ $\mathscr{P}_{t_{n}+\delta_{n}, t+\delta}^{e_{n}}(\nu)$. In all this proof, we let $C>0$ denote a generic constant which does not depend on $n$ but whose value may change from line to line.

1. We first prove that, for any fixed integer $p \geq 1$ :

$$
\lim _{n \rightarrow \infty} \mathbb{E}\left[\sup _{t \leq s \leq T}\left|X^{n}(s)-X(s)\right|^{2 p}\right]=0 .
$$

First note that $\nu^{n}=\nu$ on $[t+\delta, T]$. By using standard computations based on BurkholderDavis-Gundy's inequality, Gronwall's Lemma and the Lipschitz continuity of $b, a$ and $\beta$, we thus deduce that

$$
\mathbb{E}\left[\sup _{t+\delta \leq s \leq T}\left|X^{n}(s)-X(s)\right|^{2 p}\right] \leq C \mathbb{E}\left[\left|X^{n}(t+\delta-)-X(t+\delta-)\right|^{2 p}\right] .
$$

Since $\left(\nu^{n}, \nu\right)=(\varpi, e)$ on $\left[t_{n}+\delta_{n}, t+\delta\right)$, the Lipschitz continuity of $b$ and $a$ implies that $\mathbb{E}\left[\sup _{t_{n}+\delta_{n} \leq s<t+\delta}\left|X^{n}(s)-X(s)\right|^{2 p}\right] \leq C\left(\left|t+\delta-t_{n}-\delta_{n}\right|^{p}+\mathbb{E}\left[\left|X^{n}\left(t_{n}+\delta_{n}\right)-X\left(t_{n}+\delta_{n}\right)\right|^{2 p}\right]\right)$.

We similarly have, since $\left(\nu^{n}, \nu\right)=\left(e_{n}, e\right)$ on $\left[t, t_{n}+\delta_{n}\right)$ and $\left(X^{n}, X\right)$ does not jump at time $t_{n}+\delta_{n}$, recall Remark 2.2:

$$
\mathbb{E}\left[\sup _{t \leq s \leq t_{n}+\delta_{n}}\left|X^{n}(s)-X(s)\right|^{2 p}\right] \leq C\left(\left|e_{n}-e\right|^{2 p}+\mathbb{E}\left[\left|X^{n}(t)-x\right|^{2 p}\right]\right) .
$$

Finally, by the linear growth condition on $b$ and $a$, the fact that $\nu_{n}=e_{n}$ on $\left[t_{n}, t\right)$ and that $X^{n}$ does not jump at time $t$,

$$
\begin{aligned}
\mathbb{E}\left[\sup _{t_{n} \leq s \leq t}\left|X^{n}(s)-x\right|^{2 p}\right] & \leq C\left(\left|x_{n}-x\right|^{2 p}+\mathbb{E}\left[\sup _{t_{n} \leq s \leq t}\left|X^{n}(s)-x_{n}\right|^{2 p}\right]\right) \\
& \leq C\left(\left|x_{n}-x\right|^{2 p}+\left|t_{n}-t\right|^{p}\right) .
\end{aligned}
$$

2. We now use the above estimate to conclude the proof. We first note that

$$
\begin{aligned}
\Pi_{t_{n}, x_{n}}\left(\nu^{n}\right)-\Pi_{t, x}(\nu) & =\left(g\left(X^{n}(T)\right)-g(X(T))\right) \\
& +\left(\sum_{i \in \mathbb{I}_{t_{n}, T}^{n}} f\left(X^{n}\left(\tau_{i}^{n}+\delta_{i}^{n}-\right), \mathcal{E}_{i}^{n}\right)-\sum_{i \in \mathbb{I}_{t, T}^{\nu}} f\left(X\left(\tau_{i}^{\nu}+\delta_{i}^{\nu}-\right), \mathcal{E}_{i}^{\nu}\right)\right),
\end{aligned}
$$

with $\left(\tau^{n}, \delta^{n}, \mathcal{E}^{n}\right):=\left(\tau^{\nu^{n}}, \delta^{\nu^{n}}, \mathcal{E}^{\nu^{n}}\right)$. In view of (4.4), we can assume that

$$
\sup _{t \leq s \leq T}\left|X(s)-X^{n}(s)\right| \longrightarrow 0 \mathbb{P} \text {-a.s. }
$$

after possibly passing to a subsequence. Similar estimates show that, after possibly passing to a subsequence,

$$
\left|X^{n}\left(t_{n}+\delta_{n}-\right)-X^{n}(t+\delta-)\right| \longrightarrow 0 \mathbb{P} \text {-a.s. and in } L^{p}, p \geq 1
$$


Since $g$ is continuous, it follows that

$$
\lim _{n \rightarrow \infty} g\left(X^{n}(T)\right)=g(X(T)) \mathbb{P} \text {-a.s. }
$$

Moreover, by definition of $\nu^{n}$, we have

$$
\sum_{i \in \mathbb{H}_{t_{n}, T}^{n}} f\left(X^{n}\left(\tau_{i}^{n}+\delta_{i}^{n}-\right), \mathcal{E}_{i}^{n}\right)=f\left(X^{n}\left(t_{n}+\delta_{n}-\right), e_{n}\right)+\sum_{i \in \mathbb{I}_{t+\delta, T}^{\nu}} f\left(X^{n}\left(\tau_{i}^{\nu}+\delta_{i}^{\nu}-\right), \mathcal{E}_{i}^{\nu}\right) .
$$

It then follows from the continuity of $f,(4.6)$ and (4.7) that

$$
\begin{aligned}
\lim _{n \rightarrow \infty} \sum_{i \in \mathbb{I}_{t_{n}, T}^{\nu^{n}}} f\left(X^{n}\left(\tau_{i}^{n}+\delta_{i}^{n}-\right), \mathcal{E}_{i}^{n}\right) & =f(X(t+\delta-), e)+\sum_{i \in \mathbb{I}_{t+\delta, T}^{\nu}} f\left(X\left(\tau_{i}^{\nu}+\delta_{i}^{\nu}-\right), \mathcal{E}_{i}^{\nu}\right) \\
& =\sum_{i \in \mathbb{I}_{t, T}^{\nu}} f\left(X\left(\tau_{i}^{\nu}+\delta_{i}^{\nu}-\right), \mathcal{E}_{i}^{\nu}\right) \mathbb{P} \text {-a.s. }
\end{aligned}
$$

The required result then follows from (4.8), (4.9), (2.7), and Fatou's Lemma combined with (4.4) and (2.6) which insure that the sequence $\left(\Pi_{t_{n}, x_{n}}\left(\nu^{n}\right)^{-}\right)_{n \geq 1}$ is uniformly integrable.

We now turn to the proof of the dynamic programming principle.

Proof. [Lemma 4.1] In this proof, we consider $(\Omega, \mathcal{F}, \mathbb{F}, \mathbb{P})$ as the $d$-dimensional canonical filtered space equipped with the Wiener measure and denote by $\omega$ or $\tilde{\omega}$ a generic point. The Brownian motion is thus defined as $W(\omega)=\left(\omega_{t}\right)_{t \geq 0}$. For $\omega \in \Omega$ and $r \geq 0$, we set $\omega^{r}:=\omega . \wedge r$ and $\mathbf{T}_{r}(\omega):=\omega_{.+r}-\omega_{r}$. In the following, we omit the dependence of $\vartheta^{\nu}$ with respect to $\nu$ and simply write $\vartheta$, for ease of notations.

1. The proof of (4.1) is standard and is based on the observation that, for all $\nu \in \mathcal{S}_{t, \delta, e}^{a}$,

$$
\begin{aligned}
J(t, x ; \nu) & =\mathbb{E}\left[\mathbb{E}\left[g\left(X_{t, x}^{\nu}(T)\right)+\sum_{i \in \mathbb{I}_{\vartheta, T}^{\nu}} f\left(X_{t, x}^{\nu}\left(\tau_{i}^{\nu}+\delta_{i}^{\nu}-\right), \mathcal{E}_{i}^{\nu}\right) \mid \mathcal{F}_{\vartheta}\right]\right] \\
& +\mathbb{E}\left[\sum_{i \in \mathbb{T}_{t, \vartheta}^{\nu}} f\left(X_{t, x}^{\nu}\left(\tau_{i}^{\nu}+\delta_{i}^{\nu}-\right), \mathcal{E}_{i}^{\nu}\right)\right]
\end{aligned}
$$

where, by the flow property of $X^{\nu}$,

$$
\mathbb{E}\left[g\left(X_{t, x}^{\nu}(T)\right)+\sum_{i \in \mathbb{I}_{\vartheta, T}^{\nu}} f\left(X_{t, x}^{\nu}\left(\tau_{i}^{\nu}+\delta_{i}^{\nu}\right), \mathcal{E}_{i}^{\nu}-\right) \mid \mathcal{F}_{\vartheta}\right](\omega)=J\left(\vartheta(\omega), X_{t, x}^{\nu}(\vartheta)(\omega) ; \tilde{\nu}_{\omega}\right)
$$

with, for each $\omega \in \Omega$,

$$
\tilde{\nu}_{\omega}: \tilde{\omega} \in \Omega \mapsto \tilde{\nu}_{\omega}(\tilde{\omega})=\nu\left(\omega^{\vartheta(\omega)}+\mathbf{T}_{\vartheta(\omega)}(\tilde{\omega})\right)
$$

which can be viewed, for each $\omega \in \Omega$, as a control independent of $\mathcal{F}_{\vartheta(\omega)}$. Since the dynamic of $X_{\vartheta(\omega), X_{t, x}^{\nu}(\vartheta)(\omega)}^{\tilde{\nu}_{\omega}}$ depends on $\tilde{\nu}_{\omega}$ only through its path after $\vartheta(\omega)$, this implies that, for each $\omega \in \Omega$,

$$
\begin{aligned}
J\left(\vartheta(\omega), X_{t, x}^{\nu}(\vartheta)(\omega) ; \tilde{\nu}_{\omega}\right) & \leq \sup \left\{J\left(\vartheta(\omega), X_{t, x}^{\nu}(\vartheta)(\omega) ; \bar{\nu}\right), \bar{\nu} \in \mathcal{S}_{\vartheta(\omega), \Delta_{\vartheta}^{\nu}(\omega), \nu_{\vartheta}(\omega)}^{a}\right\} \\
& \leq\left[V^{*}, g\right]\left(\vartheta(\omega), X_{t, x}^{\nu}(\vartheta)(\omega), \Delta_{\vartheta}^{\nu}(\omega), \nu_{\vartheta}(\omega)\right),
\end{aligned}
$$


and the result follows from (4.10) and (4.11).

2. We now prove the second inequality.

2.a. We first show that, for any $\varepsilon>0$, we can find two sequences $\left(t_{n}, x_{n}, \delta_{n}, e_{n}, A_{n}\right)_{n \geq 1}$ in $D \times \mathscr{B}_{D}$ and $\left(\nu^{n}\right)_{n \geq 1}$ in $\mathcal{S}$ such that $\left(A_{n}\right)_{n \geq 1}$ forms a partition of $D$ and, for each $n$,

$\nu^{n} \in \mathcal{S}_{t_{n}, \delta_{n}, e_{n}}^{a}$ and $J\left(t^{\prime}, x^{\prime} ; \mathscr{P}_{t_{n}+\delta_{n}}^{e^{\prime}}\left(\nu^{n}\right)\right) \geq \varphi\left(t^{\prime}, x^{\prime}, \delta^{\prime}, e^{\prime}\right)-3 \varepsilon$ for all $\left(t^{\prime}, x^{\prime}, \delta^{\prime}, e^{\prime}\right) \in A_{n}$

$$
A_{n} \subset \mathcal{Q}_{r_{n}}\left(t_{n}, x_{n}, \delta_{n}, e_{n}\right) \cap D \text { for some } r_{n}>0 \text {, }
$$

where we use the notation

$$
\mathcal{Q}_{\dot{r}}(\stackrel{\circ}{t}, \stackrel{\circ}{x}, \stackrel{\circ}{\delta}, \stackrel{\circ}{e}):=\left\{\left(t^{\prime}, x^{\prime}, \delta^{\prime}, e^{\prime}\right) \in B_{\dot{r}}(\stackrel{\circ}{t}, \stackrel{\circ}{x}, \stackrel{\circ}{\dot{e}}): t^{\prime} \leq \grave{t}, t^{\prime}+\delta^{\prime} \leq \grave{t}+\stackrel{\circ}{\delta}\right\} .
$$

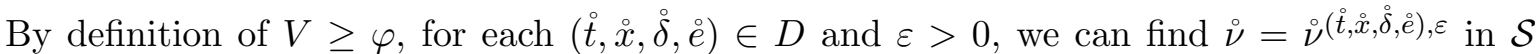
such that

$$
\stackrel{\circ}{\nu} \in \mathcal{S}_{\dot{t}, \dot{\delta}, \stackrel{\circ}{e}}^{a} \text { and } J(\stackrel{\circ}{t}, \stackrel{x}{x} ; \stackrel{\circ}{\nu}) \geq V(\stackrel{\circ}{t}, \stackrel{\circ}{x}, \stackrel{\circ}{\delta}, \stackrel{\circ}{e})-\varepsilon \geq \varphi(\stackrel{\circ}{t}, \stackrel{\circ}{x}, \stackrel{\circ}{\delta}, \stackrel{\circ}{e})-\varepsilon .
$$

Moreover, it follows from Lemma 4.2 and the upper-semicontinuity of $\varphi$ that we can find $\stackrel{r}{r}=\stackrel{\circ}{r}_{(\dot{t}, \grave{x}, \stackrel{\circ}{\delta}, \stackrel{e}{e})}$ in $(0, \infty)$ such that

$$
\begin{aligned}
J\left(t^{\prime}, x^{\prime} ; \mathscr{P}_{\grave{t}+\stackrel{\delta}{\delta}}^{e^{\prime}}(\stackrel{\circ}{\nu})\right) & \geq J(\stackrel{\circ}{t}, \stackrel{\circ}{x} ; \stackrel{\circ}{\nu})-\varepsilon \text { and } \varphi(\stackrel{\circ}{t}, \stackrel{\circ}{x}, \stackrel{\circ}{\delta}, \stackrel{\circ}{e}) \geq \varphi\left(t^{\prime}, x^{\prime}, \delta^{\prime}, e^{\prime}\right)-\varepsilon \\
& \text { for all }\left(t^{\prime}, x^{\prime}, \delta^{\prime}, e^{\prime}\right) \in \mathcal{Q}_{\dot{r}}(\stackrel{\circ}{t}, \stackrel{\circ}{x}, \stackrel{\circ}{\delta}, \stackrel{\circ}{e}) .
\end{aligned}
$$

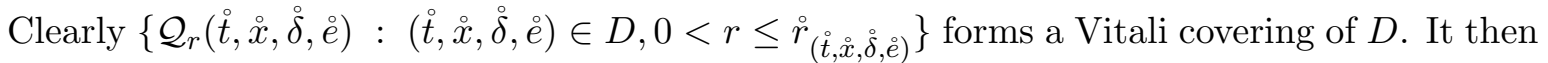
follows from the Vitali's covering Theorem, see e.g. Lemma 1.9 p10 in [9], that we can find a countable sequence $\left(t_{n}, x_{n}, \delta_{n}, e_{n}, r_{n}\right)_{n \geq 1}$ of elements of $D \times \mathbb{R}$, with $0<r_{n}<\stackrel{\circ}{r}_{\left(t_{n}, x_{n}, \delta_{n}, e_{n}\right)}$ for all $n \geq 1$, such that $D \subset \cup_{n \geq 1} \mathcal{Q}_{r_{n}}\left(t_{n}, x_{n}, \delta_{n}, e_{n}\right)$. We finally construct the sequence $\left(A_{n}\right)_{n \geq 1}$ by setting $A_{1}:=\mathcal{Q}_{r_{1}}\left(t_{1}, x_{1}, e_{1}, \delta_{1}\right) \cap D, C_{0}=\emptyset$ and

$$
A_{n}:=\left(\mathcal{Q}_{r_{n}}\left(t_{n}, x_{n}, \delta_{n}, e_{n}\right) \cap D\right) \backslash C_{n-1}, C_{n-1}:=C_{n-2} \cup A_{n-1} \quad \text { for } n \geq 2 .
$$

The sequence $\left(\nu^{n}\right)_{n \geq 1}$ is defined by $\nu^{n}:=\iota^{\left(t_{n}, x_{n}, \delta_{n}, e_{n}\right), \varepsilon}$ for all $n$.

2.b. We are now in position to prove (4.2). Let $\nu$ be a arbitrary element of $\mathcal{S}_{t, \delta, e}^{a}$ and define

$$
\hat{\nu}:=\nu \mathbf{1}_{[0, \vartheta)}+\mathbf{1}_{[\vartheta, T]} \sum_{n \geq 1} \mathscr{P}_{\vartheta+\Delta_{\vartheta}^{\nu}, t_{n}+\delta_{n}}^{\nu}\left(\nu^{n}\right) \mathbf{1}_{\left(\vartheta, X_{t, x}^{\nu}(\vartheta), \Delta_{\vartheta}^{\nu}, \nu_{\vartheta}\right) \in A_{n}} .
$$

Since $\nu \in \mathcal{S}_{t, \delta, e}^{a}$, we have $\left(\vartheta, X_{t, x}^{\nu}(\vartheta), \Delta_{\vartheta}^{\nu}, \nu_{\vartheta}\right) \in D=\cup_{n \geq 1} A_{n}$. Moreover, on $\left\{\left(\vartheta, X_{t, x}^{\nu}(\vartheta), \Delta_{\vartheta}^{\nu}, \nu_{\vartheta}\right) \in\right.$ $\left.A_{n}\right\}$, we have $\vartheta+\Delta_{\vartheta}^{\nu} \leq t_{n}+\delta_{n}$. It follows that $\hat{\nu} \in \mathcal{S}_{t, \delta, e}^{a}$, and therefore

$$
\begin{aligned}
V(t, x, \delta, e) & \geq J(t, x ; \hat{\nu}) \\
& =\mathbb{E}\left[g\left(X_{t, x}^{\hat{\nu}}(T)\right)+\sum_{i \in \mathbb{I}_{\vartheta}^{\hat{\nu}}} f\left(X_{t, x}^{\hat{\nu}}\left(\tau_{i}^{\hat{\nu}}+\delta_{i}^{\hat{\nu}}-\right), \mathcal{E}_{i}^{\hat{\nu}}\right)+\sum_{i \in \mathbb{\mathbb { H }}_{t, \vartheta}^{\hat{\nu}}} f\left(X_{t, x}^{\hat{\nu}}\left(\tau_{i}^{\hat{\nu}}+\delta_{i}^{\hat{\nu}}-\right), \mathcal{E}_{i}^{\hat{\nu}}\right)\right],
\end{aligned}
$$

where, by the flow property of $X^{\hat{\nu}}$, the fact that $\nu^{n}$ is independent of $\mathcal{F}_{t_{n}}$ and that $\vartheta \leq t_{n}$ on $\left\{\left(\vartheta, X_{t, x}^{\nu}(\vartheta), \Delta_{\vartheta}^{\nu}, \nu_{\vartheta}\right) \in A_{n}\right\}$,

$$
\mathbb{E}\left[g\left(X_{t, x}^{\hat{v}}(T)\right)\right]=\iint g\left(X_{\vartheta(\omega), X_{t, x}^{\nu}(\vartheta)(\omega)}^{\tilde{\nu}_{\omega}(\tilde{\omega})}(T)(\tilde{\omega})\right) d \mathbb{P}(\tilde{\omega}) d \mathbb{P}(\omega)
$$


and

$$
\begin{aligned}
& \mathbb{E}\left[\sum_{i \in \mathbb{I}_{\vartheta}^{\hat{\nu}}, T} f\left(X_{t, x}^{\hat{\nu}_{1}}\left(\tau_{i}^{\hat{\nu}}+\delta_{i}^{\hat{\nu}}-\right), \mathcal{E}_{i}^{\hat{\nu}}\right)\right] \\
& =\iint \sum_{i \in \mathbb{I}_{\vartheta(\omega), T}^{\tilde{\nu}_{\omega}(\tilde{\omega})}} f\left(X_{\vartheta(\omega), X_{t, x}^{\nu}}^{\tilde{\nu}_{\omega}(\tilde{\omega})(\omega)}\left(\left(\tau_{i}^{\tilde{\nu}_{\omega}}+\delta_{i}^{\tilde{\nu}_{\omega}}-\right) \wedge T\right)(\tilde{\omega}), \mathcal{E}_{i}^{\tilde{\nu}_{\omega}}(\tilde{\omega})\right) d \mathbb{P}(\tilde{\omega}) d \mathbb{P}(\omega)
\end{aligned}
$$

where, for $\omega \in \Omega$,

$\tilde{\nu}_{\omega}: \tilde{\omega} \in \Omega \mapsto \nu(\omega) \mathbf{1}_{[0, \vartheta(\omega))}+\mathbf{1}_{[\vartheta(\omega), T]} \sum_{n \geq 1} \mathscr{P}_{\vartheta(\omega)+\Delta_{\vartheta}^{\nu}(\omega), t_{n}+\delta_{n}}\left(\nu^{n}(\tilde{\omega})\right) \mathbf{1}_{\left(\vartheta(\omega), X_{t, x}^{\nu}(\vartheta)(\omega), \Delta_{\vartheta}^{\nu}(\omega), \nu_{\vartheta}(\omega)\right) \in A_{n}}$.

Hence, (4.12) implies that

$$
\begin{aligned}
V(t, x, \delta, e) & \geq \mathbb{E}\left[J\left(\vartheta, X_{t, x}^{\hat{v}}(\vartheta) ; \hat{\nu}\right)+\sum_{i \in \mathbb{\mathbb { H }}_{t, \vartheta}^{\hat{\nu}}} f\left(X_{t, x}^{\hat{\nu}}\left(\tau_{i}^{\hat{\nu}}+\delta_{i}^{\hat{\nu}}-\right), \mathcal{E}_{i}^{\hat{\nu}}\right)\right] \\
& =\mathbb{E}\left[\sum_{n \geq 1} J\left(\vartheta, X_{t, x}^{\nu}(\vartheta) ; \mathscr{P}_{\vartheta+\Delta_{\vartheta}^{\nu}, t_{n}+\delta_{n}}^{\nu_{\vartheta}}\left(\nu^{n}\right)\right) \mathbf{1}_{\left.\left(\vartheta, X_{t, x}^{\nu}(\vartheta), \Delta_{\vartheta}^{\nu}, \nu_{\vartheta}\right) \in A_{n}\right]}\right] \\
& +\mathbb{E}\left[\sum_{i \in \mathbb{T}_{t, \vartheta}^{\nu}} f\left(X_{t, x}^{\nu}\left(\tau_{i}^{\nu}+\delta_{i}^{\nu}-\right), \mathcal{E}_{i}^{\nu}\right)\right] \\
& \geq \mathbb{E}\left[\varphi\left(\vartheta, X_{t, x}^{\nu}(\vartheta), \Delta_{\vartheta}^{\nu}, \nu_{\vartheta}\right)+\sum_{i \in \mathbb{T}_{t, \vartheta}^{\nu}} f\left(X_{t, x}^{\nu}\left(\tau_{i}^{\nu}+\delta_{i}^{\nu}-\right), \mathcal{E}_{i}^{\nu}\right)\right]-3 \varepsilon
\end{aligned}
$$

By arbitrariness of $\varepsilon>0$ and $\nu \in \mathcal{S}_{t, \delta, e}^{a}$, this proves the required inequality.

Remark 4.3. Note that, by replacing $\varphi$ in (4.2) by a sequence $\left(\varphi_{k}\right)_{k \geq 1}$ of upper semicontinuous functions satisfying

$$
\varphi_{k} \leq V \text { and } \varphi_{k} \nearrow\left[V_{*}, g\right] \text { on } D,
$$

we can deduce a stronger version of (4.2):

$$
\sup _{\nu \in \mathcal{S}_{t, \delta, e}^{a}} \mathbb{E}\left[\left[V_{*}, g\right]\left(\vartheta^{\nu}, X_{t, x}^{\nu}\left(\vartheta^{\nu}\right), \Delta_{\vartheta^{\nu}}^{\nu}, \nu_{\vartheta^{\nu}}\right)+\sum_{i \in \mathbb{I}_{t, \vartheta}^{\nu}} f\left(X_{t, x}^{\nu}\left(\tau_{i}^{\nu}+\delta_{i}^{\nu}-\right), \mathcal{E}_{i}^{\nu}\right)\right] \leq V(t, x, \delta, e),
$$

where $\left[V_{*}, g\right](s, \cdot):=V_{*}(s, \cdot) \mathbf{1}_{s<T}+g \mathbf{1}_{s=T}$. In particular, if $V$ is continuous, combining (4.1) and the previous inequality leads to the classical version of the dynamic programming principle (3.1).

\subsection{Viscosity properties}

Now we are in position to prove Theorem 3.2. We split the proof in different propositions. 


\subsubsection{Supersolution property}

We start with the supersolution property in the domain $D=D_{E,>0} \cup D_{\varpi}$.

Proposition 4.4. The function $V_{*}$ is a viscosity supersolution of (3.2) on D.

Proof. The proof follows from standard arguments except that we use the non classical formulation of the dynamic programming principle (4.2). Fix $\left(t_{0}, x_{0}, \delta_{0}, e_{0}\right) \in D$ and let $\varphi$ be a smooth function such that $\left(t_{0}, x_{0}, \delta_{0}, e_{0}\right)$ achieves a (global) minimum of $V_{*}-\varphi$ on $D$ such that

$$
0=\left(V_{*}-\varphi\right)\left(t_{0}, x_{0}, \delta_{0}, e_{0}\right) .
$$

Let $\left(t_{k}, x_{k}, \delta_{k}, e_{k}\right)_{k \geq 1}$ be a sequence in $D$ such that

$$
\left(t_{k}, x_{k}, \delta_{k}, e_{k}\right) \longrightarrow\left(t_{0}, x_{0}, \delta_{0}, e_{0}\right) \text { and } V\left(t_{k}, x_{k}, \delta_{k}, e_{k}\right) \longrightarrow V_{*}\left(t_{0}, x_{0}, \delta_{0}, e_{0}\right) \text { as } k \longrightarrow \infty,
$$

and observe that

$$
(\varphi-V)\left(t_{k}, x_{k}, \delta_{k}, e_{k}\right) \longrightarrow 0 \text { when } k \longrightarrow \infty \text {. }
$$

Case 1. We first assume that $\left(t_{0}, x_{0}, \delta_{0}, e_{0}\right) \in D_{E,>0}$ and that

$$
-\mathcal{L}^{e_{0}} \varphi\left(t_{0}, x_{0}, \delta_{0}, e_{0}\right)+\frac{\partial}{\partial \delta} \varphi\left(t_{0}, x_{0}, \delta_{0}, e_{0}\right)=:-2 \varepsilon<0,
$$

and work towards a contradiction. Define the function $\bar{\varphi}$ by

$$
\bar{\varphi}(t, x, \delta, e):=\varphi(t, x, \delta, e)-\left|x-x_{0}\right|^{4}-\left|t-t_{0}\right|^{2}-\left|\delta-\delta_{0}\right|^{2},
$$

so that $\bar{\varphi}$ also satisfies (4.17). By continuitiy of $b$ and $a$, we can find $r>0$ such that $(4.19)\left(-\mathcal{L}^{e} \bar{\varphi}+\frac{\partial}{\partial \delta} \bar{\varphi}\right)(t, x, \delta, e) \leq 0$ for $(t, x, \delta, e) \in B:=B_{r}\left(t_{0}, x_{0}, \delta_{0}, e_{0}\right) \cap D_{E,>0}$.

Given $k$ large enough so that $\left(t_{k}, x_{k}, \delta_{k}, e_{k}\right) \in B$, let $\nu^{k}$ be any control in $\mathcal{S}_{t_{k}, \delta_{k}, e_{k}}^{a}$. Set $\left(X^{k}, \Delta^{k}\right):=\left(X_{t_{k}, x_{k}}^{\nu^{k}}, \Delta^{\nu^{k}}\right)$ and define

$$
\theta^{k}:=\inf \left\{s \geq t_{k}:\left(s, X^{k}(s), \Delta_{s}^{k}\right) \notin B\right\} .
$$

For $r$ small enough we have $\Delta_{\theta^{k}}^{\nu^{k}}>0$ and therefore $\nu^{k}=e_{k}$ on $\left[t_{k}, \theta^{k}\right]$. Using Itô's Lemma, (4.19) and the definition of $\bar{\varphi}$, we thus obtain that

$$
\bar{\varphi}\left(t_{k}, x_{k}, \delta_{k}, e_{k}\right) \leq \mathbb{E}\left[\bar{\varphi}\left(\theta^{k}, X^{k}\left(\theta^{k}\right), \Delta_{\theta^{k}}^{k}, e_{k}\right)\right] \leq \mathbb{E}\left[\varphi\left(\theta^{k}, X^{k}\left(\theta^{k}\right), \Delta_{\theta^{k}}^{k}, \nu_{\theta^{k}}^{k}\right)\right]-\eta
$$

where $\eta:=\inf \left\{\left|x-x_{0}\right|^{4}+\left|t-t_{0}\right|^{2}+\left|\delta-\delta_{0}\right|^{2},\left(t, x, \delta, e_{k}\right) \in \partial B_{r}\left(t_{0}, x_{0}, \delta_{0}, e_{0}\right)\right\}>0$, observe that $\left|e_{k}-e_{0}\right|<r$. Since $I_{t_{k}, \theta^{k}}^{\nu^{k}}=\emptyset$, the above inequality combined with (4.16) and (4.18) contradict (4.2) for $k$ large enough.

Case 2. We now assume that $\left(t_{0}, x_{0}, \delta_{0}, e_{0}\right) \in D_{\varpi}$. Since $E$ is closed and $\varpi \notin E,(4.15)$ implies that

$$
\left(\delta_{k}, e_{k}\right)=(0, \varpi) \text { for } k \text { large enough. }
$$


We now assume that

$$
\min \left\{-\mathcal{L}^{\varpi} \varphi\left(t_{0}, x_{0}, 0, \varpi\right), \varphi\left(t_{0}, x_{0}, 0, \varpi\right)-\mathcal{M}[\varphi]\left(t_{0}, x_{0}\right)\right\}=:-2 \varepsilon<0,
$$

and work toward a contradiction. If

$$
-\mathcal{L}^{\varpi} \varphi\left(t_{0}, x_{0}, 0, \varpi\right)=-2 \varepsilon<0,
$$

we can argue as above to obtain a contradiction to (4.2). If

$$
\varphi\left(t_{0}, x_{0}, 0, \varpi\right)-\mathcal{M}[\varphi]\left(t_{0}, x_{0}\right)=:-2 \varepsilon<0,
$$

we can find $(\hat{\delta}, \hat{e}) \in\left[\underline{\delta}, T-t_{0}\right) \times E$ and $r>0$ such that

$$
\varphi(t, x, 0, \varpi)-\varphi(t, x+\beta(x, \hat{e}, \hat{\delta}), \hat{\delta}, \hat{e}) \leq-\varepsilon, \text { for }(t, x) \in B:=B_{r}\left(t_{0}, x_{0}\right) .
$$

Let $\bar{\nu}$ denote the constant control that takes the value $\varpi$ on $[0, T]$, set $\bar{X}^{k}:=X_{t_{k}, x_{k}}^{\bar{\nu}}$ and

$$
\theta^{k}:=\inf \left\{s \geq t_{k}:\left(s, \bar{X}^{k}(s)\right) \notin B\right\} \wedge\left(t_{k}+k^{-1}\right) .
$$

Note that for $k$ large enough, we have $t_{k}+k^{-1}+\hat{\delta} \leq T$. We can then define $\nu^{k} \in \mathcal{S}_{t_{k}, 0, \varpi}^{a}$ by

$$
\nu_{t}^{k}:=\hat{e} \mathbf{1}_{t \in\left[\theta^{k}, \theta^{k}+\hat{\delta}\right)}+\varpi \mathbf{1}_{t \notin\left[\theta^{k}, \theta^{k}+\hat{\delta}\right)}, t \leq T,
$$

and set $\left(X^{k}, \Delta^{k}\right):=\left(X_{t_{k}, x_{k}}^{\nu^{k}}, \Delta^{\nu^{k}}\right)$. Using Itô's Lemma and (4.22), we obtain that

$$
\varphi\left(t_{k}, x_{k}, 0, \varpi\right) \leq \mathbb{E}\left[\varphi\left(\theta^{k}, X^{k}\left(\theta^{k}\right), \Delta_{\theta^{k}}^{k}, \nu_{\theta^{k}}^{k}\right)-\varepsilon\right]+C / k,
$$

for some $C>0$ which does not depend on $k$. The above inequality combined with (4.16) contradict (4.2) for $k$ large enough.

We now turn to the proof of the boundary conditions.

Proposition 4.5. Fix $\left(t_{0}, x_{0}, \delta_{0}, e_{0}\right) \in \bar{D}$. Then,

$$
V_{*}\left(t_{0}, x_{0}, 0, e_{0}\right) \geq\left\{\begin{array}{lll}
V_{*}\left(t_{0}, x_{0}, 0, \varpi\right)+f\left(x_{0}, e_{0}\right) & \text { if } \quad\left(t_{0}, x_{0}, \delta_{0}, e_{0}\right) \in D_{E, 0} \\
\mathcal{V}\left(t_{0}, x_{0}, e_{0}\right) & \text { if } \quad\left(t_{0}, x_{0}, \delta_{0}, e_{0}\right) \in D_{E, T} \\
g\left(x_{0}\right)+f\left(x_{0}, e_{0}\right) & \text { if } \quad\left(t_{0}, x_{0}, \delta_{0}, e_{0}\right) \in D_{T} .
\end{array}\right.
$$

Proof. We only prove the first inequality. The two other ones follow from similar arguments. Let $\left(t_{k}, x_{k}, \delta_{k}, e_{k}\right)_{k \geq 1}$ be a sequence in $D$ such that

(4.23) $\left(t_{k}, x_{k}, \delta_{k}, e_{k}\right) \longrightarrow\left(t_{0}, x_{0}, 0, e_{0}\right)$ and $V\left(t_{k}, x_{k}, \delta_{k}, e_{k}\right) \longrightarrow V_{*}\left(t_{0}, x_{0}, 0, e_{0}\right)$ as $k \longrightarrow \infty$.

For each $k \geq 1$, define

$$
\nu^{k}:=\varpi \mathbf{1}_{\left[0, t_{k}+\delta_{k}\right)}+e_{k} \mathbf{1}_{\left[0, t_{k}+\delta_{k}\right)} \in \mathcal{S}_{t_{k}, \delta_{k}, e_{k}}^{a},
$$

and set $X^{k}:=X_{t_{k}, x_{k}}^{\nu^{k}}$. It follows from Remark 4.3 that

$$
V\left(t_{k}, x_{k}, \delta_{k}, e_{k}\right) \geq \mathbb{E}\left[V_{*}\left(t_{k}+\delta_{k}, X^{k}\left(t_{k}+\delta_{k}\right), 0, \varpi\right)+f\left(X^{k}\left(t_{k}+\delta_{k}-\right), e_{k}\right)\right], k \geq 1 .
$$

Using standard estimates, see e.g. the proof of Lemma 4.2, one easily checks that $X^{k}\left(t_{k}+\right.$ $\left.\delta_{k}-\right) \rightarrow x_{0}$ in $L^{p}$ for all $p \geq 1$, and in particular $\mathbb{P}$-a.s., after possibly passing to a subsequence. It thus follows from the lower-semicontinuity of $V_{*}$ and $f$ that, up to a subsequence,

$\liminf _{k \rightarrow \infty} V_{*}\left(t_{k}+\delta_{k}, X^{k}\left(t_{k}+\delta_{k}\right), 0, \varpi\right)+f\left(X^{k}\left(t_{k}+\delta_{k}-\right), e_{k}\right) \geq V_{*}\left(t_{0}, x_{0}, 0, \varpi\right)+f\left(x_{0}, e_{0}\right) \mathbb{P}$-a.s.

The required result then follows from (4.23), (4.24), and the last inequality combined with polynomial growth property of $f$ and $V$, see Remark 2.4, and Fatou's Lemma. 


\subsubsection{Subsolution property}

We start with the subsolution property in the domain $D=D_{E,>0} \cup D_{\varpi}$.

Proposition 4.6. The function $V^{*}$ is a viscosity subsolution of (3.2) on D.

Proof. Fix $\left(t_{0}, x_{0}, \delta_{0}, e_{0}\right) \in D$ and let $\varphi$ be a smooth function such that $\left(t_{0}, x_{0}, \delta_{0}, e_{0}\right)$ achieves a (global) maximum of $V^{*}-\varphi$ such that

$$
0=\left(V^{*}-\varphi\right)\left(t_{0}, x_{0}, \delta_{0}, e_{0}\right) .
$$

In the following, we denote by $\left(t_{k}, x_{k}, \delta_{k}, e_{k}\right)_{k \geq 1}$ a sequence in $D$ satisfying

$$
\left(t_{k}, x_{k}, \delta_{k}, e_{k}\right) \longrightarrow\left(t_{0}, x_{0}, \delta_{0}, e_{0}\right) \text { and } V\left(t_{k}, x_{k}, \delta_{k}, e_{k}\right) \longrightarrow V^{*}\left(t_{0}, x_{0}, \delta_{0}, e_{0}\right) \text { as } k \longrightarrow \infty .
$$

Case 1. We first assume that $\left(t_{0}, x_{0}, \delta_{0}, e_{0}\right) \in D_{E,>0}$ and that

$$
-\mathcal{L}^{e_{0}} \varphi\left(t_{0}, x_{0}, \delta_{0}, e_{0}\right)+\frac{\partial}{\partial \delta} \varphi\left(t_{0}, x_{0}, \delta_{0}, e_{0}\right)=: 2 \varepsilon>0,
$$

and work towards a contradiction. By continuity of $b$ and $a$, we can find $r>0$ such that

$$
\left(-\mathcal{L}^{e} \varphi+\frac{\partial}{\partial \delta} \varphi\right)(t, x, \delta, e) \geq \varepsilon \quad \text { for }(t, x, \delta, e) \in B:=B_{r}\left(t_{0}, x_{0}, \delta_{0}, e_{0}\right) \cap D_{E,>0} .
$$

Moreover, we can always assume that $\left(t_{0}, x_{0}, \delta_{0}, e_{0}\right)$ achieves a strict local maximum, so that after possibly changing the value of $\varepsilon$, we have

$$
\sup _{\partial_{p} B}\left(V^{*}-\varphi\right)=:-\varepsilon<0,
$$

where $\partial_{p} B$ is the parabolic boundary of $B$. Fix $\nu^{k} \in \mathcal{S}_{t_{k}, \delta_{k}, e_{k}}$ and set

$$
\theta^{k}:=\inf \left\{s \geq t_{k}:\left(s, X^{k}(s), \Delta_{s}^{k}, \nu_{s}^{k}\right) \notin B\right\},
$$

where $\left(X^{k}, \Delta^{k}\right):=\left(X_{t_{k}, x_{k}}^{\nu^{k}}, \Delta^{\nu^{k}}\right)$. Observe that, for $r$ small enough, $\Delta_{\theta^{k}}^{k}>0$ and therefore $\nu^{k}=e_{k}$ on $\left[t_{k}, \theta^{k}\right]$. Applying Itô's Lemma to $\varphi$ and using (4.26) and (4.27), we deduce that

$$
\varphi\left(t_{k}, x_{k}, \delta_{k}, e_{k}\right) \geq \mathbb{E}\left[\varphi\left(\theta^{k}, X^{k}\left(\theta^{k}\right), \Delta_{\theta^{k}}^{k}, \nu_{\theta^{k}}\right)\right] \geq \mathbb{E}\left[V^{*}\left(\theta^{k}, X^{k}\left(\theta^{k}\right), \Delta_{\theta^{k}}^{k}, \nu_{\theta^{k}}\right)\right]+\varepsilon .
$$

Since $\mathbb{I}_{t_{k}, \theta^{k}}^{\nu^{k}}=\emptyset$, this contradicts (4.1) for $k$ large enough, recall (4.25) .

Case 2. We now assume that $\left(t_{0}, x_{0}, \delta_{0}, e_{0}\right)=\left(t_{0}, x_{0}, 0, \varpi\right) \in D_{\varpi}$ and

$$
\min \left\{-\mathcal{L}^{\varpi} \varphi\left(t_{0}, x_{0}, 0, \varpi\right), \varphi\left(t_{0}, x_{0}, 0, \varpi\right)-\mathcal{M}[\varphi]\left(t_{0}, x_{0}\right)\right\}=: 2 \varepsilon>0,
$$

and work towards a contradiction. By continuity of $b$ and $a$, we can find $r>0$ such that

$$
\min \left\{-\mathcal{L}^{\varpi} \varphi(\cdot, 0, \varpi), \varphi(\cdot, 0, \varpi)-\mathcal{M}[\varphi]\right\} \geq \varepsilon \text { on } B:=B_{r}\left(t_{0}, x_{0}\right) \cap\left([0, T) \times \mathbb{R}^{d}\right) .
$$

Moreover, without loss of generality we can assume that $\left(t_{0}, x_{0}\right)$ achieves a strict local maximum, so that after possibly changing the value of $\varepsilon$

$$
\sup _{\partial_{p} B}\left(V^{*}(\cdot, 0, \varpi)-\varphi(\cdot, 0, \varpi)\right)=:-\varepsilon<0,
$$


where $\partial_{p} B$ is the parabolic boundary of $B$. Also observe that, since $E$ is closed and $\varpi \notin E$, (4.25) implies that

$$
\left(\delta_{k}, e_{k}\right)=(0, \varpi) \text { for } k \text { large enough. }
$$

Let $\nu^{k} \in \mathcal{S}_{t_{k}, 0, \varpi}^{a}=\mathcal{S}_{t_{k}, \delta_{k}, e_{k}}^{a}$ be arbitrary, set $\left(X^{k}, \Delta^{k},\left(\tau_{i}^{k}\right)_{i \geq 1}\right):=\left(X_{t_{k}, x_{k}}^{\nu^{k}}, \Delta^{\nu^{k}},\left(\tau_{i}^{\nu^{k}}\right)_{i \geq 1}\right)$ and define

$$
\theta^{k}:=\inf \left\{s \geq t_{k}:\left(s, X^{k}(s)\right) \notin B\right\}, \vartheta^{k}:=\inf \left\{\tau_{i}^{k}, i \geq 1 \text { s.t. } \tau_{i}^{k} \geq t_{k}\right\} \text { and } \xi^{k}:=\theta^{k} \wedge \vartheta^{k} .
$$

Applying Itô's Lemma to $\varphi$, using (4.28), (4.29), and recalling (4.30) lead to

$$
\begin{aligned}
\varphi\left(t_{k}, x_{k}, \delta_{k}, e_{k}\right) & \geq \mathbb{E}\left[\varphi\left(\xi^{k}, X^{k}\left(\xi^{k}-\right), 0, \varpi\right)\right] \\
& \geq \mathbb{E}\left[\varphi\left(\xi^{k}, X^{k}\left(\xi^{k}\right), \Delta_{\xi^{k}}^{k}, \nu_{\xi^{k}}^{k}\right)+\varepsilon \mathbf{1}_{\xi^{k}=\vartheta^{k}}\right] \\
& \geq \mathbb{E}\left[V^{*}\left(\xi^{k}, X^{k}\left(\xi^{k}\right), \Delta_{\xi^{k}}^{k}, \nu_{\xi^{k}}^{k}\right)\right]+\varepsilon .
\end{aligned}
$$

In view of (4.25) this leads to a contradiction with (4.1) for $k$ large enough.

We now turn to the boundary condition $\delta \rightarrow 0$.

Proposition 4.7. For all $\left(t_{0}, x_{0}, \delta_{0}, e_{0}\right) \in D_{E, 0}$, we have

$$
V^{*}\left(t_{0}, x_{0}, 0, e_{0}\right) \leq V^{*}\left(t_{0}, x_{0}, 0, \varpi\right)+f\left(x_{0}, e_{0}\right) .
$$

Proof. By following similar arguments as in the second step of the proof of Proposition 4.6 above, one easily checks that, for any smooth function $\bar{\varphi}$ such that $\left(t_{0}, x_{0}, 0, e_{0}\right)$ achieves a global maximum of $V^{*}-\bar{\varphi}$ satisfying $\left(V^{*}-\bar{\varphi}\right)\left(t_{0}, x_{0}, 0, e_{0}\right)=0$, we have

$$
\min \left\{-\mathcal{L}^{e_{0}} \varphi\left(t_{0}, x_{0}, 0, e_{0}\right)+\frac{\partial}{\partial \delta} \bar{\varphi}\left(t_{0}, x_{0}, 0, e_{0}\right), \bar{\varphi}\left(t_{0}, x_{0}, 0, e_{0}\right)-\bar{\varphi}\left(t_{0}, x_{0}, 0, \varpi\right)-f\left(x_{0}, e_{0}\right)\right\} \leq 0 .
$$

Let now $\varphi$ be a smooth function such that $\left(t_{0}, x_{0}, 0, e_{0}\right)$ achieves a global maximum of $V^{*}-\varphi$ satisfying $\left(V^{*}-\varphi\right)\left(t_{0}, x_{0}, 0, e_{0}\right)=0$, and consider the function $\bar{\varphi}$ defined as

$$
\bar{\varphi}_{\varepsilon}(t, x, \delta, e):=\varphi(t, x, \delta, e)+\sqrt{\varepsilon+\delta}-\sqrt{\varepsilon}
$$

for some $\varepsilon>0$. Observe that $\left(t_{0}, x_{0}, 0, e_{0}\right)$ achieves a global maximum of $V^{*}-\bar{\varphi}_{\varepsilon}$. It thus follows that either

$$
\bar{\varphi}_{\varepsilon}\left(t_{0}, x_{0}, 0, e_{0}\right)-\bar{\varphi}_{\varepsilon}\left(t_{0}, x_{0}, 0, \varpi\right)-f\left(x_{0}, e_{0}\right) \leq 0
$$

or

$$
-\mathcal{L}^{e_{0}} \varphi\left(t_{0}, x_{0}, 0, e_{0}\right)+\frac{\partial}{\partial \delta} \varphi\left(t_{0}, x_{0}, 0, e_{0}\right)+\varepsilon^{-\frac{1}{2}} \leq 0 .
$$

Clearly, the second assertion can not hold for $\varepsilon>0$ small enough. It follows that

$$
\bar{\varphi}_{\varepsilon}\left(t_{0}, x_{0}, 0, e_{0}\right)-\bar{\varphi}_{\varepsilon}\left(t_{0}, x_{0}, 0, \varpi\right)-f\left(x_{0}, e_{0}\right) \leq 0
$$

for all $\varepsilon>0$ small enough, which provides the required result.

We next consider the boundary conditions as $t+\delta \rightarrow T$. 
Proposition 4.8. For all $\left(t_{0}, x_{0}, \delta_{0}, e_{0}\right) \in D_{E, T}$, we have

$$
V^{*}\left(t_{0}, x_{0}, \delta_{0}, e_{0}\right) \leq \mathcal{V}\left(t_{0}, x_{0}, e_{0}\right) .
$$

Proof. Let $\left(t_{k}, x_{k}, \delta_{k}, e_{k}\right)_{k \geq 1}$ be a sequence in $D$ satisfying

$$
\left(t_{k}, x_{k}, \delta_{k}, e_{k}\right) \longrightarrow\left(t_{0}, x_{0}, \delta_{0}, e_{0}\right) \text { and } V\left(t_{k}, x_{k}, \delta_{k}, e_{k}\right) \longrightarrow V^{*}\left(t_{0}, x_{0}, \delta_{0}, e_{0}\right) \text { as } k \longrightarrow \infty .
$$

For $k$ large enough, we have $t_{k}+\delta_{k}>T-\underline{\delta}$ so that, for any $\nu^{k} \in \mathcal{S}_{t_{k}, \delta_{k}, e_{k}}^{a}$, we have $\nu^{k}=e_{k}$ on $\left[t_{k}, t_{k}+\delta_{k}\right)$ and $\nu^{k}=\varpi$ on $\left[t_{k}+\delta_{k}, T\right]$, recall (2.1). It follows that

$$
V\left(t_{k}, x_{k}, \delta_{k}, e_{k}\right)=\mathbb{E}\left[g\left(X_{t_{k}, x_{k}}^{\nu^{k}}(T)\right)+f\left(\mathcal{X}_{t_{k}, x_{k}}^{e_{k}}\left(t_{k}+\delta_{k}\right), e_{k}\right)\right],
$$

recall the definition of $\mathcal{X}$ in (2.10). Moreover, since $t_{k}+\delta_{k} \rightarrow t_{0}+\delta_{0}=T$, standard estimates based on (2.5) and Remark 2.2 imply that $\left(X_{t_{k}, x_{k}}^{\nu^{k}}(T), \mathcal{X}_{t_{k}, x_{k}}^{e_{k}}\left(t_{k}+\delta_{k}\right)\right) \rightarrow\left(\mathcal{X}_{t_{0}, x_{0}}^{e_{0}}(T), \mathcal{X}_{t_{0}, x_{0}}^{e_{0}}\left(t_{0}+\right.\right.$ $\left.\left.\delta_{0}\right)\right)$ as $k \rightarrow \infty$ in $L^{p}$ for all $p \geq 1$. The result then follows by taking the limsup as $k \rightarrow \infty$ in the above equality and using (2.7) as well as the dominated convergence theorem.

We finally discuss the boundary condition at $t=T$.

Proposition 4.9. For all $\left(T, x_{0}, \delta_{0}, e_{0}\right) \in D_{T}$, we have

$$
V^{*}\left(T, x_{0}, \delta_{0}, e_{0}\right) \leq g\left(x_{0}\right)+f\left(x_{0}, e\right) .
$$

Proof. This is proved by similar arguments as in the proof of Proposition 4.8 above.

\subsection{A comparison result}

In this section, we provide the proof of Theorem 3.3. We first show that (3.2) admits a classical strict supersolution in the following sense:

Proposition 4.10. For any integer $p \geq \gamma$, there exists a function $\Lambda$ on $\mathbb{R}^{d} \times \mathbb{R}_{+} \times \bar{E}$ and $\varrho>0$ satisfying

(i) $\Lambda \in C^{2,1,0}\left(\mathbb{R}^{d} \times \mathbb{R}_{+} \times \bar{E}\right)$,

(ii) $\Lambda \geq g^{+}+f^{+}+\mathcal{V}^{+}$,

(iii) $\inf _{(\delta, e) \in[0, T] \times \bar{E}} \Lambda(x, \delta, e) /|x|^{p} \rightarrow \infty$ as $|x| \rightarrow \infty$,

(iv) $\langle b, D \Lambda\rangle+\frac{1}{2} \operatorname{Tr}\left[a a^{*} D^{2} \Lambda\right] \leq \varrho \Lambda$ and $-\partial \Lambda / \partial \delta+\langle b, D \Lambda\rangle+\frac{1}{2} \operatorname{Tr}\left[a a^{*} D^{2} \Lambda\right] \leq \varrho \Lambda$ on $\mathbb{R}^{d} \times \mathbb{R}_{+} \times \bar{E}$,

(v) $\Lambda(x, 0, e)-f(x, e)-q(x) \geq \Lambda(x, 0, \varpi) \geq \Lambda(x+\beta(x, e, \delta), \delta, e)+\underline{\delta}$ for all $x \in \mathbb{R}^{d}, \delta \in[\underline{\delta}, T]$ and $e \in E$, where $q$ is a continuous and (strictly) positive function on $\mathbb{R}^{d}$.

Proof. Let $\varphi$ be a $C^{1}\left(\mathbb{R}_{+}\right)$function with bounded first derivative such that $\varphi \geq 0, \varphi(0)=1$ and $\varphi(\delta)=0$ for $\delta \geq \underline{\delta}$ and let $\Lambda$ be defined by:

$$
\Lambda(x, \delta, e)=\mu\left(1+|x|^{2 p}\right)\left(1+\kappa \mathbf{1}_{e=\varpi}\right)+\left(2 \kappa \mu\left(1+|x|^{2 p}\right)-\delta\right) \varphi(\delta) \mathbf{1}_{e \neq \varpi}
$$

for some $\kappa>0$ and $\mu>T$ such that $f(x, e)^{+}+g(x)^{+}+\mathcal{V}(t, x, e)^{+} \leq \mu\left(1+|x|^{2 p}\right)$, recall (2.7) and Remark 2.4. The first three assertions clearly hold for $\mu$ large enough, recall that 
$\varpi \notin E$ where $E$ is closed. As for the fourth assertion, we recall that $b$ and $a$ are uniformly Lipschitz, so that the left-hand side is of order $\left(1+|x|^{2 p}\right)$, which is dominated by $\varrho \Lambda$ for $\varrho$ large enough. The right-hand side inequality holds for $\varrho$ large enough by similar arguments. Finally, recalling (2.5), we observe that, for $\mu$ and $\kappa$ large enough,

$$
\begin{aligned}
\Lambda(x, 0, e)-f(x, e)-\Lambda(x, 0, \varpi) & =\kappa \mu\left(1+|x|^{2 p}\right)-f(x, e) \geq \mu\left(1+|x|^{2 p}\right), \\
\Lambda(x, 0, \varpi)-\Lambda(x+\beta(x, e, \delta), \delta, e) & =\mu\left(1+|x|^{2 p}\right)(1+\kappa)-\mu\left(1+|x+\beta(x, e, \delta)|^{2 p}\right) \\
& \geq \mu \kappa / 2 \geq \underline{\delta}
\end{aligned}
$$

for all $x \in \mathbb{R}^{d}, \underline{\delta} \leq \delta \leq T$ and $e \in E$, which provides the last assertion for $q(x):=\mu(1+$ $\left.|x|^{2 p}\right)$.

We can now provide the proof of Theorem 3.3.

Proof. [Theorem 3.3] Let $u$ and $v$ be as in Theorem 3.3 and let $p \geq \gamma$ be large enough so that

$$
[v(t, x, \delta, e)-u(t, x, \delta, e)]^{+} \leq C\left(1+|x|^{p}\right) \text { on } \bar{D}
$$

for some $C>0$. We assume that

$$
\sup _{\bar{D}}(v-u) \geq 2 \eta \text { for some } \eta>0
$$

and work toward a contradiction.

1. Let $\varrho>0$ and $\Lambda$ be as in Proposition 4.10 for $p \geq \gamma$ satisfying (4.31). It follows from (4.32) that for $\lambda \in(0,1)$ small enough there is some $\left(t_{\lambda}, x_{\lambda}, \delta_{\lambda}, e_{\lambda}\right) \in \bar{D}$ such that

$$
\max _{\bar{D}}(\tilde{v}-\tilde{w})=(\tilde{v}-\tilde{w})\left(t_{\lambda}, x_{\lambda}, \delta_{\lambda}, e_{\lambda}\right) \geq \eta>0
$$

where for a map $\varphi$ on $\bar{D}$, we write $\tilde{\varphi}(t, x, \delta, e)$ for $e^{\varrho t} \varphi(t, x, \delta, e)$, and $w:=(1-\lambda) u+\lambda \Lambda$. Observe that $\tilde{u}, \tilde{v}$ are super- and subsolution of

$$
\tilde{\mathcal{H}} \varphi=0
$$

on $\bar{D}$, where, for a smooth function $\varphi$ defined on $\bar{D}$,

$$
\tilde{\mathcal{H}} \varphi(t, x, \delta, e):=\left\{\begin{array}{ccc}
\left(\varrho \frac{\partial}{\partial t}-\mathcal{L}^{e}+\frac{\partial}{\partial \delta}\right) \varphi(t, x, \delta, e) & \text { on } & D_{E,>0}, \\
\varphi(t, x, \delta, e)-\varphi(t, x, 0, \varpi)-\tilde{f}(x, e) & \text { on } & D_{E, 0}, \\
\varphi(t, x, \delta, e)-\tilde{\mathcal{V}}(t, x, e) & \text { on } & D_{E, T}, \\
\min \left\{\left(\varrho \frac{\partial}{\partial t}-\mathcal{L}^{\varpi}\right) \varphi(t, x, \delta, e) ; \varphi(t, x, \delta, e)-\mathcal{M}[\varphi](t, x)\right\} & \text { on } & D_{\varpi}, \\
\varphi(t, x, \delta, e)-\tilde{g}(x)-\tilde{f}(x, e) & \text { on } & D_{T} .
\end{array}\right.
$$

Also note that

$$
\left(t_{\lambda}, x_{\lambda}, \delta_{\lambda}, e_{\lambda}\right) \notin D_{E, T} \cup D_{T}
$$

since otherwise the super- and subsolution property of $u$ and $v$ would imply

$$
(v-w)\left(t_{\lambda}, x_{\lambda}, \delta_{\lambda}, e_{\lambda}\right) \leq \lambda\left(\mathcal{V}\left(t_{\lambda}, x_{\lambda}, e_{\lambda}\right) \vee\left(g\left(x_{\lambda}\right)+f\left(x_{\lambda}, e_{\lambda}\right)\right)-\Lambda\left(t_{\lambda}, x_{\lambda}, \delta_{\lambda}, e_{\lambda}\right)\right)
$$

which, in view of (ii) in Proposition 4.10, would contradict (4.33). 
2. For $(t, x, \delta, e) \in \bar{D}$ and $n \geq 1$, we now set

$$
\begin{aligned}
\Gamma(t, x, y, \delta, e) & :=\tilde{v}(t, x, \delta, e)-\tilde{w}(t, y, \delta, e) \\
\Theta_{n}(t, x, y, \delta, e) & :=\Gamma(t, x, y, \delta, e)-\varphi_{n}(t, x, y, \delta, e)
\end{aligned}
$$

where

$$
\varphi_{n}(t, x, y, \delta, e):=n|x-y|^{2 p}+\left|x-x_{\lambda}\right|^{2 p+2}+\left|t-t_{\lambda}\right|^{2}+\left|\delta-\delta_{\lambda}\right|^{2}+\left|e-e_{\lambda}\right| .
$$

By the growth assumption on $v, u$ and the fact that $\bar{E}$ is compact, there is $\left(t_{n}, x_{n}, y_{n}, \delta_{n}, e_{n}\right) \in$ $\bar{D}^{2}$, with

$$
\bar{D}^{2}:=\{(t, x, y, \delta, e):((t, x, \delta, e),(t, y, \delta, e)) \in \bar{D} \times \bar{D}\}
$$

such that

$$
\max _{\bar{D}^{2}} \Theta_{n}=\Theta_{n}\left(t_{n}, x_{n}, y_{n}, \delta_{n}, e_{n}\right)
$$

Since

$$
\Gamma\left(t_{n}, x_{n}, y_{n}, \delta_{n}, e_{n}\right) \geq \Theta_{n}\left(t_{n}, x_{n}, y_{n}, \delta_{n}, e_{n}\right) \geq(\tilde{v}-\tilde{w})\left(t_{\lambda}, x_{\lambda}, \delta_{\lambda}, e_{\lambda}\right)
$$

it follows from the growth condition on $v$ and $u$, (iii) of Proposition 4.10 and the uppersemicontinuity of $\Lambda$ that, up to a subsequence,

$$
\begin{aligned}
\left(t_{n}, x_{n}, y_{n}, \delta_{n}, e_{n}\right) & \longrightarrow\left(t_{\lambda}, x_{\lambda}, x_{\lambda}, \delta_{\lambda}, e_{\lambda}\right) \\
\varphi_{n}\left(t_{n}, x_{n}, y_{n}, \delta_{n}, e_{n}\right) & \longrightarrow 0 \\
\Gamma\left(t_{n}, x_{n}, y_{n}, \delta_{n}, e_{n}\right) & \longrightarrow \Gamma\left(t_{\lambda}, x_{\lambda}, x_{\lambda}, \delta_{\lambda}, e_{\lambda}\right)
\end{aligned}
$$

3. It follows from (4.36) and (4.37) that, after possibly passing to a subsequence,

$$
\left(t_{n}, x_{n}, \delta_{n}, e_{n}\right) \notin D_{E, T} \cup D_{T} \text { for all } n \geq 1 .
$$

3.1. We now assume that, up to a subsequence, $e_{n} \neq \varpi$ for all $n \geq 1$.

If $\left|\left\{n: \delta_{n}=0\right\}\right|=\infty$, then we can assume that, up to a subsequence, $\delta_{n}=0$, i.e., $\left(t_{n}, x_{n}, \delta_{n}, e_{n}\right) \in D_{E, 0}$ for all $n \geq 1$. It then follows from the super- and subsolution property of $\tilde{u}$ and $\tilde{v}$, and (v) of Proposition 4.10, that

$$
\begin{aligned}
\tilde{v}\left(t_{n}, x_{n}, 0, e_{n}\right) & \leq \tilde{v}\left(t_{n}, x_{n}, 0, \varpi\right)+\tilde{f}\left(x_{n}, e_{n}\right) \\
\tilde{u}\left(t_{n}, y_{n}, 0, e_{n}\right) & \geq \tilde{u}\left(t_{n}, y_{n}, 0, \varpi\right)+\tilde{f}\left(y_{n}, e_{n}\right) \\
\tilde{\Lambda}\left(y_{n}, 0, e_{n}\right) & \geq \tilde{\Lambda}\left(y_{n}, 0, \varpi\right)+\tilde{f}\left(y_{n}, e_{n}\right)+e^{\varrho t_{n}} q\left(y_{n}\right),
\end{aligned}
$$

and therefore

$$
\Gamma\left(t_{n}, x_{n}, y_{n}, 0, e_{n}\right) \leq \Gamma\left(t_{n}, x_{n}, y_{n}, 0, \varpi\right)+\left(\tilde{f}\left(x_{n}, e_{n}\right)-\tilde{f}\left(y_{n}, e_{n}\right)\right)-\lambda e^{\varrho t_{n}} q\left(y_{n}\right) .
$$

Sending $n \rightarrow \infty$ and using (4.37) and (4.39) leads to

$$
\Gamma\left(t_{\lambda}, x_{\lambda}, x_{\lambda}, \delta_{\lambda}, e_{\lambda}\right) \leq \Gamma\left(t_{\lambda}, x_{\lambda}, x_{\lambda}, 0, \varpi\right)-\lambda e^{\varrho t_{\lambda}} q\left(y_{\lambda}\right)
$$

which, recalling that $q>0$ on $\mathbb{R}^{d}$, contradicts (4.33). 
It follows from the above arguments that $\left|\left\{n: \delta_{n}=0\right\}\right|<\infty$. In this case, $\left(t_{n}, x_{n}, \delta_{n}, e_{n}\right) \in$ $D_{E,>0}$ for all $n \geq 1$ large enough, recall (4.40). Using the viscosity property of $\tilde{u}$ and $\tilde{v}$, (iv) in Proposition 4.10, and standard arguments based on Ishii's Lemma, see [8], together with (4.37) allows to deduce that

$$
\varrho \Gamma\left(t_{n}, x_{n}, y_{n}, \delta_{n}, e_{n}\right) \leq O_{\lambda}\left(n^{-1}\right),
$$

where $O_{\lambda}\left(n^{-1}\right) \rightarrow 0$ as $n \rightarrow \infty$. Since $\varrho>0$, combining the above inequality with (4.39) leads to a contradiction to (4.33).

3.2. We now assume that, up to a subsequence, $e_{n}=\varpi$, so that $\left(t_{n}, x_{n}, \delta_{n}, e_{n}\right) \in D_{\varpi}$ for all $n \geq 1$. Note that we can not have

$$
\tilde{v}\left(t_{n}, x_{n}, 0, \varpi\right)-\sup _{(\delta, e) \in\left[\underline{\delta}, T-t_{n}\right] \times E} \tilde{v}\left(t_{n}, x_{n}, \delta, e\right) \leq 0
$$

along a subsequence, since otherwise the supersolution property of $\tilde{u}$ and (v) of Proposition 4.10 would imply

$$
\Gamma\left(t_{n}, x_{n}, y_{n}, 0, \varpi\right) \leq \sup _{(\delta, e) \in\left[\underline{\delta}, T-t_{n}\right] \times E} \Gamma\left(t_{n}, x_{n}, y_{n}, \delta, e\right)-\lambda e^{\varrho t_{n}} \underline{\delta},
$$

which would contradict (4.33) for $n$ large enough, recall (4.37), (4.39) and the fact that $\underline{\delta}>0$. We can thus assume that $\tilde{v}\left(t_{n}, x_{n}, 0, \varpi\right)-\sup _{(\delta, e) \in\left[\underline{\delta}, T-t_{n}\right] \times E} \tilde{v}\left(t_{n}, x_{n}, \delta, e\right)>0$ for $n$ large enough. Using again the viscosity properties of $\tilde{u}$ and $\tilde{v}$, (iv) of Proposition 4.10, standard arguments based on Ishii's Lemma, see [8], and (4.37) then leads

$$
\varrho \Gamma\left(t_{n}, x_{n}, y_{n}, \delta_{n}, e_{n}\right) \leq O_{\lambda}\left(n^{-1}\right)
$$

where $O_{\lambda}\left(n^{-1}\right) \rightarrow 0$ as $n \rightarrow \infty$. As above, this leads to a contradiction.

\section{Numerical approximation}

In this section, we construct a finite difference scheme to solve the PDE (3.2) numerically, and prove the convergence of the numerical scheme.

\subsection{Space discretization}

Given a positive integer $N$, we discretize the set

$$
\mathcal{T}:=\left\{(t, \delta) \in[0, T] \times \mathbb{R}_{+}: \underline{\delta} \leq t+\delta \leq T\right\}
$$

in

$$
\mathcal{T}_{N}:=\left\{i h_{N} \wedge T,(i+j) h_{N}, i=0, \ldots, N \text { and } j=0, \ldots, N-i\right\}
$$

where

$$
h_{N}:=T / N .
$$

We next fix a positive integer $M$ and $R>0$ and approximate $\mathcal{R}_{R}^{d}:=B_{R}(0) \subset \mathbb{R}^{d}$ by

$$
\mathcal{R}_{M R}^{d}:=\left\{-R+k h_{M R}, k=0, \ldots, 2 M\right\}^{d}
$$


where

$$
h_{M R}:=R / M \text {. }
$$

We finally consider an increasing sequence $\left(E_{L}\right)_{L \geq 1}$ of finite subsets of $E$ such that

$$
\cup_{L \geq 1} E_{L}=E .
$$

For ease of notations, we set

$$
\bar{D}_{N M}^{R L}:=\left\{(t, x, \delta, e) \in \bar{D}:(t, \delta) \in \mathcal{T}_{N}, x \in \mathcal{R}_{M R}^{d}, e \in E_{L} \cup\{\varpi\}\right\} .
$$

\section{$5.2 \quad$ Finite difference approximation}

From now on, we denote by $x^{i}$ the $i$-th component of a vector $x \in \mathbb{R}^{d}$, and by $A^{i j}$ the $(i, j)$ component of a matrix $A \in \mathbb{M}^{d}$. We use the notation $l_{i}$ for the unit vector of $\mathbb{R}^{d}$ in the $i^{t h}$ coordinate direction.

We use the standard finite difference approximation, see [13] for a full description.

Space component:

$$
\begin{gathered}
\frac{\partial \psi}{\partial x^{i}}(t, x, \delta, e) \sim\left\{\begin{array}{l}
\frac{\psi\left(t, x+h_{M R} l_{i}, \delta, e\right)-\psi(t, x, \delta, e)}{h_{M R}} \equiv \Delta_{x^{i}}^{h_{M R^{+}}} \psi(t, x, \delta, e) \text { if } b^{i}(x, e) \geq 0 \\
\frac{\psi(t, x, \delta, e)-\psi\left(t, x-h_{M R} l_{i}, \delta, e\right)}{h_{M R}} \equiv \Delta_{x^{i}}^{h_{M R^{-}}} \psi(t, x, \delta, e) \text { if } b^{i}(x, e)<0
\end{array}\right. \\
\frac{\partial^{2} \psi}{\partial x_{i}^{2}}(t, x, \delta, e) \sim \frac{\psi\left(t, x+h_{M R} l_{i}, \delta, e\right)-2 \psi(t, x, \delta, e)+\psi\left(t, x-h_{M R} l_{i}, \delta, e\right)}{h_{M R}^{2}} \equiv \Delta_{x^{i} x^{i}}^{h_{M R}} \psi(t, x, \delta, e) .
\end{gathered}
$$

If $a^{i j}(x, e) \geq 0, i \neq j$, then

$$
\begin{aligned}
\frac{\partial^{2} \psi}{\partial x^{i} x^{j}}(t, x, \delta, e) \sim & \frac{2 \psi(t, x, \delta, e)+\psi\left(t, x+h_{M R}\left(l_{i}+l_{j}\right), \delta, e\right)+\psi\left(t, x-h_{M R}\left(l_{i}+l_{j}\right), \delta, e\right)}{h_{M R}^{2}} \\
& -\frac{\psi\left(t, x+h_{M R} l_{i}, \delta, e\right)+\psi\left(t, x-h_{M R} l_{i}, \delta, e\right)}{2 h_{M R}^{2}} \\
& -\frac{\psi\left(t, x+h_{M R} l_{j}, \delta, e\right)+\psi\left(t, x-h_{M R} l_{j}, \delta, e\right)}{2 h_{M R}^{2}} \\
\equiv & \Delta_{x^{i} x^{j}}^{h_{M R}+} \psi(t, x, \delta, e) .
\end{aligned}
$$

If $a^{i j}(x, e)<0, i \neq j$, then

$$
\begin{aligned}
\frac{\partial^{2} \psi}{\partial x^{i} x^{j}}(t, x, \delta, e) \sim & -\frac{\left[2 \psi(t, x, \delta, e)+\psi\left(t, x+h_{M R}\left(l_{i}-l_{j}\right), \delta, e\right)+\psi\left(t, x-h_{M R}\left(l_{i}-l_{j}\right), \delta, e\right)\right]}{h_{M R}^{2}} \\
& +\frac{\psi\left(t, x+h_{M R} l_{i}, \delta, e\right)+\psi\left(t, x-h_{M R} l_{i}, \delta, e\right)}{2 h_{M R}^{2}} \\
& +\frac{\psi\left(t, x+h_{M R} l_{j}, \delta, e\right)+\psi\left(t, x-h_{M R} l_{j}, \delta, e\right)}{2 h_{M R}^{2}} \\
\equiv & \Delta_{x^{i} x^{j}}^{h_{M R}} \psi(t, x, \delta, e) .
\end{aligned}
$$


For ease of notations we write:

$$
\Delta_{x}^{h_{M R}} \psi(t, x, \delta, e):=\left(\Delta_{x^{i}}^{h_{M R}+} \psi(t, x, \delta, e) \mathbf{1}_{b^{i}(x, e) \geq 0}+\Delta_{x^{i}}^{h_{M R^{-}}} \psi(t, x, \delta, e) \mathbf{1}_{b^{i}(x, e)<0}\right)_{i \leq d} \in \mathbb{R}^{d}
$$

and

$$
\Delta_{x x}^{h_{M R}} \psi:=\left(\Delta_{x^{i} x^{j}}^{h_{M R}+} \psi(t, x, \delta, e) \mathbf{1}_{a^{i j}(x, e) \geq 0}+\Delta_{x^{i} x^{j}}^{h_{M R^{-}}} \psi(t, x, \delta, e) \mathbf{1}_{a^{i j}(x, e)<0}\right)_{i, j \leq d} \in \mathbb{M}^{d} .
$$

Time component:

$$
\frac{\partial \psi}{\partial t}(t, x, \delta, e) \sim \frac{\psi\left(t+h_{N}, x, \delta, e\right)-\psi(t, x, \delta, e)}{h_{N}} \equiv \Delta_{t}^{h_{N}} \psi(t, x, \delta, e) .
$$

Latency duration:

$$
\frac{\partial \psi}{\partial \delta}(t, x, \delta, e) \sim \frac{\psi(t, x, \delta, e)-\psi\left(t, x, \delta-h_{N}, e\right)}{h_{N}} \equiv \Delta_{\delta}^{h_{N}} \psi(t, x, \delta, e) .
$$

\subsection{Approximation scheme of (3.2) and convergence}

We now define $\tilde{V}_{N M}^{R}$ as the solution on $\bar{D}_{N M}^{R L}$ of:

$$
\mathcal{H}_{N M}^{R L} \varphi(t, x, \delta, e) \mathbf{1}_{x \notin \partial \mathcal{R}_{R}^{d}}+(\varphi(t, x, \delta, e)-g(x)) \mathbf{1}_{x \in \partial \mathcal{R}_{R}^{d}}=0
$$

where $\mathcal{H}_{N M}^{R L} \varphi(t, x, \delta, e)$ is given by

$$
\left\{\begin{array}{cll}
\left(-\mathcal{L}_{N M}^{R, e}+\Delta_{\delta}^{h_{N}}\right) \varphi(t, x, \delta, e) & \text { on } & \bar{D}_{N M}^{R L} \cap D_{E,>0}, \\
\varphi(t, x, \delta, e)-\varphi\left(t+h_{N}, x, 0, \varpi\right)-f(x, e) & \text { on } & \bar{D}_{N M}^{R L} \cap D_{E, 0}, \\
\varphi(t, x, \delta, e)-\mathcal{V}(t, x, e) & \text { on } & \bar{D}_{N L}^{R L} \cap D_{E, T}, \\
\min \left\{-\mathcal{L}_{N M}^{\varpi} \varphi(t, x, \delta, e), \varphi(t, x, \delta, e)-\mathcal{M}_{L M}^{R}[\varphi](t, x)\right\} & \text { on } & \bar{D}_{N M}^{R L} \cap D_{\varpi}, \\
\varphi(t, x, \delta, e)-g(x)-f(x, e) & \text { on } & \bar{D}_{N L}^{R L} \cap D_{T},
\end{array}\right.
$$

with

$$
\begin{aligned}
\mathcal{L}_{M N}^{R, e} \varphi(t, x, \delta, e) & :=\Delta_{t}^{h_{N}} \varphi(t, x, \delta, e)+\left\langle b(x, e), \Delta_{x}^{h_{M R}} \varphi(t, x, \delta, e)\right\rangle+\frac{1}{2} \operatorname{Tr}\left[a a^{*}(x, e) \Delta_{x x}^{h_{M R}} \varphi(t, x)\right] . \\
\mathcal{M}_{L M}^{R}[\varphi](t, x) & := \\
\left\{\delta^{\prime}, e^{\prime}\right) \in\left([\delta, T-t] \cap\left\{i h_{N}\right\}_{1 \leq i \leq N}\right) \times E_{L} & \varphi\left(t, \Pi_{M}^{R}\left(x+\beta\left(x, e^{\prime}, \delta^{\prime}\right)\right), \delta^{\prime}, e^{\prime}\right) .
\end{aligned}
$$

Here, $\Pi_{M}^{R}$ denotes the projection operator on $\mathcal{R}_{M R}^{d}$

$$
\Pi_{M}^{R}(x):=\left(\left[\left(-R \vee x^{i} \wedge R\right) / M\right] M\right)_{i \leq d},
$$

with [·] denoting the integer part.

From now on, we write $\tilde{V}_{n}$ for $\tilde{V}_{N_{n} M_{n}}^{R_{n} L_{n}}$ where $\left(N_{n}, M_{n}, R_{n}, L_{n}\right)_{n \geq 1}$ is a sequence of positive integers such that

$$
N_{n}, M_{n}, R_{n}, L_{n} \uparrow \infty \text { as } n \rightarrow \infty,
$$

and we denote by $\bar{V}$ and $\underline{V}$ the relaxed semilimits of $\tilde{V}_{n}$ :

$$
\begin{aligned}
& \bar{V}(t, x, \delta, e):=\lim _{\substack{\left(i_{h} h_{N}, i_{x} h_{M R}, i_{\delta} h_{N}, e^{\prime}\right) \in \bar{D} \rightarrow(t, x, \delta, e) \\
n \rightarrow \infty}} \tilde{V}_{n}\left(i_{h} h_{N}, i_{x} h_{M R}, i_{\delta} h_{N}, e^{\prime}\right) \\
& \underline{V}(t, x, \delta, e):=\quad \liminf _{\substack{\left(i_{h} h_{N}, i_{x} h_{M R}, i_{\delta} h_{N}, e^{\prime}\right) \in \bar{D} \rightarrow(t, x, \delta, e) \\
n \rightarrow \infty}} \tilde{V}_{n}\left(i_{h} h_{N}, i_{x} h_{M R}, i_{\delta} h_{N}, e^{\prime}\right) .
\end{aligned}
$$


One easily checks that the above scheme is monotone, in the terminology of [5]. Moreover, recalling (2.7) and (2.8), easy computations based on a induction argument also lead to the following uniform polynomial control on $\bar{V}$ and $\underline{V}$ under the additional classical condition:

$$
h_{M R}^{2}=h_{N} .
$$

Proposition 5.1. The above scheme is monotone. If the condition (5.2), then there exists a constant $C>0$, independent on $N, M, L$ and $R$, such that

$$
|\bar{V}(t, x, \delta, e)|+|\underline{V}(t, x, \delta, e)| \leq C\left(1+|x|^{\gamma}\right) \text { on } \bar{D} .
$$

Using the fact that $f(\cdot, \varpi)=0$ and $\mathcal{V}(T, \cdot)=g+f$, recall (2.7) and Remark 2.5, we now observe that, if a function $\varphi$ satisfies

$$
\max \{\varphi-\mathcal{V}, \varphi-\varphi(\cdot, 0, \varpi)-f, \varphi-g-f\} \mathbf{1}_{e \neq \varpi}+(\varphi-g-f) \mathbf{1}_{e=\varpi} \geq 0 \text { on } D_{T},
$$

then it also satisfies

$$
\varphi-g-f \geq 0 \text { on } D_{T}
$$

Similarly, if it satisfies

$$
\min \{\varphi-\mathcal{V}, \varphi-\varphi(\cdot, 0, \varpi)-f, \varphi-g-f\} \mathbf{1}_{e \neq \varpi}+(\varphi-g-f) \mathbf{1}_{e=\varpi} \leq 0 \text { on } D_{T},
$$

then it also satisfies

$$
\varphi-g-f \leq 0 \text { on } D_{T} .
$$

It then follows from the arguments of [5], and the continuity of $f, g$ and $\mathcal{V}$, see Remark 2.5, that $\underline{V}$ is a supersolution on $\bar{D}$ of $\mathcal{H}^{*} \varphi=0$ and that $\bar{V}$ is a subsolution on $\bar{D}$ of $\mathcal{H}_{*} \varphi=0$ where

$$
\mathcal{H}^{*} \varphi:=\left\{\begin{array}{ccc}
H_{E,>0} \varphi:=\left(-\mathcal{L}^{e}+\frac{\partial}{\partial \delta}\right) \varphi & \text { on } & D_{E,>0}, \\
H_{\varpi} \varphi:=\max \left\{-\mathcal{L}^{\varpi} \varphi ; \varphi-\mathcal{M}[\varphi]\right\} & \text { on } & D_{\varpi}, \\
\max \left\{H_{E,>0} \varphi, \varphi-\varphi(\cdot, 0, \varpi)-f\right\} & \text { on } & D_{E, 0}, \\
\max \left\{H_{E,>0} \varphi, \varphi-\mathcal{V}\right\} & \text { on } & D_{E, T}, \\
\max \left\{H_{E,>0} \varphi \mathbf{1}_{E}+H_{\varpi} \varphi \mathbf{1}_{\{\varpi\}}, \varphi-g-f\right\} & \text { on } & D_{T} .
\end{array}\right.
$$

and

$$
\mathcal{H}_{*} \varphi:=\left\{\begin{array}{ccc}
H_{E,>0} \varphi:=\left(-\mathcal{L}^{e}+\frac{\partial}{\partial \delta}\right) \varphi & \text { on } & D_{E,>0} \\
H_{\varpi} \varphi:=\min \left\{-\mathcal{L}^{\varpi} \varphi ; \varphi-\mathcal{M}[\varphi]\right\} & \text { on } & D_{\varpi}, \\
\min \left\{H_{E,>0} \varphi, \varphi-\varphi(\cdot, 0, \varpi)-f\right\} & \text { on } & D_{E, 0}, \\
\min \left\{H_{E,>0} \varphi, \varphi-\mathcal{V}\right\} & \text { on } & D_{E, T}, \\
\min \left\{H_{E,>0} \varphi \mathbf{1}_{E}+H_{\varpi} \varphi \mathbf{1}_{\{\varpi\}}, \varphi-g-f\right\} & \text { on } & D_{T} .
\end{array}\right.
$$

In order to conclude that $\underline{V}=\bar{V}=V$ on $\bar{D}$, it remains to prove the following result.

Proposition 5.2. Let $\psi$ be lower-semicontinuous function with polynomial growth. If $\psi$ is a viscosity supersolution (resp. subsolution) of $\mathcal{H}^{*} \varphi=0$ (resp. $\mathcal{H}_{*} \varphi=0$ ) on $\bar{D}$, then $\psi$ is a viscosity supersolution (resp. subsolution) of $\mathcal{H} \varphi=0$. 
Proof. We only prove the supersolution property, the subsolution property being proved by similar arguments. Let $\psi$ be a supersolution of $\mathcal{H}^{*} \varphi=0$. Let $\left(t_{0}, x_{0}, \delta_{0}, e_{0}\right) \in \bar{D}$ and let $\varphi$ be a smooth function such that $\left(t_{0}, x_{0}, \delta_{0}, e_{0}\right)$ achieves a (global) minimum of $\psi-\varphi$ satisfying $(\psi-\varphi)\left(t_{0}, x_{0}, \delta_{0}, e_{0}\right)=0$. If $\left(t_{0}, x_{0}, \delta_{0}, e_{0}\right) \in D_{E,>0} \cup D_{\varpi}$ then $\mathcal{H} \varphi\left(t_{0}, x_{0}, \delta_{0}, e_{0}\right) \geq 0$. If $\left(t_{0}, x_{0}, \delta_{0}, e_{0}\right) \in D_{E, 0} \cup D_{T}$, then similar arguments as in the proof of Proposition 4.7 shows that $\mathcal{H} \varphi\left(t_{0}, x_{0}, \delta_{0}, e_{0}\right) \geq 0$ too.

It remains to study the case where $\left(t_{0}, x_{0}, \delta_{0}, e_{0}\right) \in D_{E, T}$. We claim that, the map $(t, x) \in$ $[0, T) \times \mathbb{R}^{d} \rightarrow \psi\left(t, x, T-t, e_{0}\right)$ is a supersolution of

$$
\max \left\{-\mathcal{L}^{e_{0}} \varphi, \varphi-\mathcal{V}\right\}\left(\cdot, e_{0}\right) \geq 0 \text { on }[0, T) \times \mathbb{R}^{d}
$$

with the terminal condition

$$
\max \{\varphi-g-f, \varphi-\mathcal{V}\}\left(T, \cdot, e_{0}\right) \geq 0 \text { on } \mathbb{R}^{d} .
$$

Since $\mathcal{V}$ is a subsolution of the same equation, recal Remark 2.5, applying a standard comparison principle (recall our Lipschitz continuity and growth assumptions and see e.g. [8]), will readily implies that $\psi \geq \mathcal{V}$.

We conclude this proof by proving the above claim. Fix $e_{0} \in E$, and let $\left(t_{0}, x_{0}\right) \in[0, T] \times \mathbb{R}^{d}$ and $\varphi$ be smooth function such that $\left(t_{0}, x_{0}\right)$ achieves a global minimum (equal to 0 ) of $(t, x) \mapsto$ $\psi\left(t, x, T-t, e_{0}\right)-\varphi(t, x)$. For $n \geq 1$, we define $\varphi_{n}$ by $\varphi_{n}(t, x, \delta, e):=\varphi(t, x)-n(T-t-\delta)-$ $\left|t-t_{0}\right|^{2 p}-\left|x-x_{0}\right|^{2 p}-\left|e-e_{0}\right|^{2 p}$, for $p \geq 1$ such that $(t, x, \delta, e) \in \bar{D} \mapsto|\psi(t, x, \delta, e)| /\left(1+|x|^{p}\right)$ is bounded. Let $\left(t_{n}, x_{n}, \delta_{n}, e_{n}\right)_{n}$ be a global minimum point of $\psi-\varphi_{n}$. Writing that

$$
\begin{aligned}
\psi\left(t_{0}, x_{0}, T-t_{0}, e_{0}\right)-\varphi\left(t_{0}, x_{0}\right) & \geq\left(\psi-\varphi_{n}\right)\left(t_{n}, x_{n}, \delta_{n}, e_{n}\right) \\
& =(\psi-\varphi)\left(t_{n}, x_{n}, \delta_{n}, e_{n}\right) \\
& +n\left(T-t_{n}-\delta_{n}\right)+\left|t_{n}-t_{0}\right|^{2 p}+\left|x_{n}-x_{0}\right|^{2 p}+\left|e_{n}-e_{0}\right|^{2 p} \\
& \geq(\psi-\varphi)\left(t_{n}, x_{n}, \delta_{n}, e_{n}\right)
\end{aligned}
$$

one easily checks that

$$
\left(t_{n}, x_{n}, e_{n}\right) \rightarrow\left(t_{0}, x_{0}, e_{0}\right), n\left(T-t_{n}-\delta_{n}\right) \rightarrow 0 \text { and } \psi\left(t_{n}, x_{n}, \delta_{n}, e_{n}\right) \rightarrow \psi\left(t_{0}, x_{0}, T-t_{0}, e_{0}\right) .
$$

Note that, since $e_{0} \in E$, we have $e_{n} \neq \varpi$ for $n$ large enough. Moreover, the supersolution property of $\psi$ implies that $\mathcal{H}^{*} \varphi_{n}\left(t_{n}, x_{n}, \delta_{n}, e_{n}\right) \geq 0$. Since $-\partial \varphi_{n} / \partial t+\partial \varphi_{n} / \partial \delta=-\partial \varphi / \partial t+$ $2 p\left(t_{n}-t_{0}\right)^{2 p-1}$, it follows from (5.5) that, for $n$ large enough,

$$
\begin{array}{cll}
-\mathcal{L}^{e_{n}} \varphi\left(t_{n}, x_{n}\right) \geq \varepsilon_{n} & \text { if } \quad & \left(t_{n}, x_{n}, \delta_{n}, e_{n}\right) \in D_{E,>0}, \\
-\mathcal{L}^{e_{n}} \varphi \vee(\varphi-\mathcal{V})\left(t_{n}, x_{n}, e_{n}\right) \geq \varepsilon_{n} & \text { if } \quad\left(t_{n}, x_{n}, \delta_{n}, e_{n}\right) \in D_{E, T}, \\
-\mathcal{L}^{e_{n}} \varphi \vee(\varphi-g-f)\left(t_{n}, x_{n}, e_{n}\right) \geq \varepsilon_{n} & \text { if } \quad\left(t_{n}, x_{n}, \delta_{n}, e_{n}\right) \in D_{T},
\end{array}
$$

where $\varepsilon_{n} \rightarrow 0$ as $n \rightarrow \infty$. Taking the limit as $n \rightarrow \infty$ and using (5.5) then implies that

$$
\max \left\{-\mathcal{L}^{e_{0}} \varphi, \varphi-\mathcal{V}\right\}\left(t_{0}, x_{0}, e_{0}\right) \geq 0 \text { if } t_{0}<T
$$

and

$$
\max \left\{-\mathcal{L}^{e_{0}} \varphi, \varphi-g-f, \varphi-\mathcal{V}\right\}\left(t_{0}, x_{0}, e_{0}\right) \geq 0 \text { if } t_{0}=T
$$

which, by similar arguments as in the proof of Proposition 4.7, implies that

$$
\max \{\varphi-g-f, \varphi-\mathcal{V}\}\left(t_{0}, x_{0}, e_{0}\right) \geq 0 \text { if } t_{0}=T .
$$


We can now conclude by using the comparison principle of Theorem 3.3, recall Proposition 5.2, Proposition 5.1, Theorem 3.2 and Remark 2.4.

Theorem 5.3. We have:

$$
\underline{V}=\bar{V}=V \text { on } \bar{D}
$$

\section{Examples of applications in algorithmic trading}

\subsection{Example 1}

As a first example, we consider the case where the aim of the controller is to sell a number $Q_{0}$ of one stock $S$ between 0 and $T>0$. We denote by $V_{t}$ the global volume instantaneously traded on the market at time $t$. The dynamics of $(S, V)$ is given by the strong solution of the SDE

$$
\begin{aligned}
S_{t} & =S_{0}+\int_{0}^{t} \mu_{S}\left(r, S_{r}, V_{r}\right) d r+\int_{0}^{t} \sigma_{S}\left(r, S_{r}, V_{r}\right) d W_{r}, \\
V_{t} & =V_{0}+\int_{0}^{t} \mu_{V}\left(r, S_{r}, V_{r}\right) d r+\int_{0}^{t} \sigma_{V}\left(r, S_{r}, V_{r}\right) d W_{r},
\end{aligned}
$$

where $W$ denotes a two dimensional standard Brownian motion, and $\left(\mu_{S}, \sigma_{S}, \mu_{V}, \sigma_{V}\right)$ are Lipschitz continuous. We implicitly assume here that the above SDE has non-negative solutions whatever the initial conditions are.

A control $\nu \in S$ is identified to a sequence $\left(\tau_{i}^{\nu}, \delta_{i}^{\nu}, \mathcal{E}_{i}^{\nu}\right)_{i \geq 1} \in \mathcal{S}$ as in Section 2. Here $\mathcal{E}_{i}^{\nu}$ stands for the proportion of the remain number of shares that have to be sold, $Q_{\tau_{i}^{\nu}}^{\nu}$, which will be traded on $\left[\tau_{i}^{\nu}, \tau_{i}^{\nu}+\delta_{i}^{\nu}\right)$. We assume that this quantity is sold uniformly on the corresponding time interval. Namely, we sell $\mathcal{E}_{i}^{\nu} Q_{\tau_{i}}^{\nu} / \delta_{i}^{\nu} d t$ on $[t, t+d t]$ for $t \in\left[\tau_{i}^{\nu}, \tau_{i}^{\nu}+\delta_{i}^{\nu}\right)$.

This means that the dynamics of the remaining number of stocks to sell is given by

$$
Q_{t}^{\nu}=Q_{0}-\sum_{i \geq 1} \int_{\tau_{i}^{\nu}}^{\tau_{i}^{\nu}+\delta_{i}^{\nu}} \mathbf{1}_{s \leq t} \mathcal{E}_{i}^{\nu} Q_{\tau_{i}^{\nu}}^{\nu} / \delta_{i}^{\nu} d s .
$$

Clearly, $E$ has to be contained in $[0,1]$.

Due to the impact of the strategy on the market, the obtained price of the executed volume under the regime $\mathcal{E}_{i}^{\nu}$ is

$$
\tilde{S}_{t}=S_{t}-\eta\left(\mathcal{E}_{i}^{\nu} Q_{\tau_{i}^{\nu}}^{\nu} / \delta_{i}^{\nu}, S_{t}, V_{t}\right), t \in\left[\tau_{i}^{\nu}, \tau_{i}^{\nu}+\delta_{i}^{\nu}\right)
$$

where $\eta$ is the market impact function and is assumed to be Lipschitz continuous. It follows that the cumulated wealth's dynamic is

$$
Y_{t}^{\nu}=0+\sum_{i \geq 1} \int_{\tau_{i}^{\nu}}^{\tau_{i}^{\nu}+\delta_{i}^{\nu}} \mathbf{1}_{r \leq t} \tilde{S}_{r} \mathcal{E}_{i}^{\nu} Q_{\tau_{i}^{\nu}}^{\nu} / \delta_{i}^{\nu} d r .
$$

The remain part $Q_{T}^{\nu}$ is instantaneously sold on the market at a price: $S_{T}-c\left(Q_{T}^{\nu}, S_{T}, V_{T}\right)$, for some Lipschitz continuous function.

The total gain after the final transaction is thus given by:

$$
Y_{T}^{\nu}+\left(S_{T}-c\left(Q_{T}^{\nu}, S_{T}, V_{T}\right)\right)\left(Q_{T}^{\nu}\right)^{+} .
$$

The aim of the controller is to maximize the expectation of the quantity

$$
g\left(Y_{T}^{\nu}+\left(S_{T}-c\left(Q_{T}^{\nu}, S_{T}, V_{T}\right)\right)\left(Q_{T}^{\nu}\right)^{+}\right)
$$

for some concave function $g$ with polynomial growth. 


\subsection{Example 2}

As a second example, we consider the case where the aim of the controller is to buy a number $Q_{0}$ of one stock $S$ between 0 and $T>0$. The dynamics of $(S, V)$ is given as in the previous example.

Here $\mathcal{E}_{i}^{\nu}$ stands for the intensity at which the stocks are bought, i.e. the algorithm buys a number $\mathcal{E}_{i}^{\nu} d t=\nu_{t} \mathbf{1}_{\nu_{t} \neq \varpi} d t$ of stocks on $[t, t+d t], t \in\left[\tau_{i}^{\nu}, \tau_{i}^{\nu}+\delta_{i}^{\nu}\right)$. We fix $E:=\left[0, E_{\text {max }}\right]$ for some $E_{\max }>0$. For ease of notations, we introduce the function $q: \bar{E} \mapsto \mathbb{R}_{+}$defined by $q(e)=e \mathbf{1}_{e \neq \varpi}$. The dynamics of the remaining number of stocks to be bought before $T$ is thus given by:

$$
Q_{t}^{\nu}=Q_{0}-\int_{0}^{t} q\left(\nu_{s}\right) d s
$$

Due to the impact of the strategy on the market, the obtained price of the executed volume under the regime $e$ is

$$
\tilde{S}_{t}=S_{t}+\eta\left(e, S_{t}, V_{t}\right)
$$

where $\eta$ is the market impact function which depends on the global volume instantaneously traded on the market $V_{t}$, and is assumed to be Lipschitz continuous. It follows that the cumulated wealth's dynamic is

$$
Y_{t}^{\nu}=0+\int_{0}^{t} \tilde{S}_{r} q\left(\nu_{r}\right) d r=0+\int_{0}^{t}\left(S_{r}+\eta\left(\nu_{r}, S_{r}, V_{r}\right)\right) q\left(\nu_{r}\right) d r .
$$

If the number $Q_{0}$ of shares is not liquidated at time $T$, the remain part $Q_{T}^{\nu}$ is instantaneously bought on the market at a price: $S_{T}+c\left(Q_{T}^{\nu}, S_{T}, V_{T}\right)$, for some Lipschitz continuous function. The total cost after the final transaction is thus given by:

$$
Y_{T}^{\nu}+\left(S_{T}+c\left(Q_{T}^{\nu}, S_{T}, V_{T}\right)\right)\left(Q_{T}^{\nu}\right)^{+} .
$$

The aim of the controller is to minimize the expectation of the quantity

$$
\ell\left(Y_{T}^{\nu}+\left(S_{T}+c\left(Q_{T}^{\nu}, S_{T}, V_{T}\right)\right)\left(Q_{T}^{\nu}\right)^{+}\right)
$$

for some convex function $\ell$ with polynomial growth.

As an example of application, we provide here a numerical study of the impact of the instantaneous market volume on the optimal strategy. We consider the following set of parameters. The trading period corresponds to one day of 7 hours, i.e. 240 minutes, which we discretize in 150 steps of size $\Delta t$. The price process is assumed to follow a Black and Scholes dynamics $S_{t}=S_{0} e^{-\frac{1}{2} \sigma^{2} t+\sigma W_{t}}$, where $S_{0}:=2.18$ and $\sigma=0.06$, which corresponds to an annual volatility of $20 \%$. We assume a deterministic evolution of the instantaneous volume traded on the market $\left(V_{t}\right)_{t \leq T}$. The impact function $\eta$ is given by $\eta(e, v)=0.03(e / v)^{1.1}$. We take $\underline{\delta}=24$ minutes. The set of regimes is the interval $[0,34]$ which we discretize in 30 equidistant steps. This corresponds to a maximal impact of approximately $4 \%$ of the initial stock value $S_{0}$. The final cost is given by $c(q, v)=0.03(q /(v \Delta t))^{1.1}$. The fact that $v$ is multiplied by $\Delta t$ in the last expression comes from the fact the last order is executed in a single step and not at a rate $q$ on a time interval of length $\Delta t$. We simply consider the case where $\ell$ is the identity. In this case, the value function is clearly linear in the $y$-variable which allows for simplifications in the numerical resolution of the associated PDEs. 

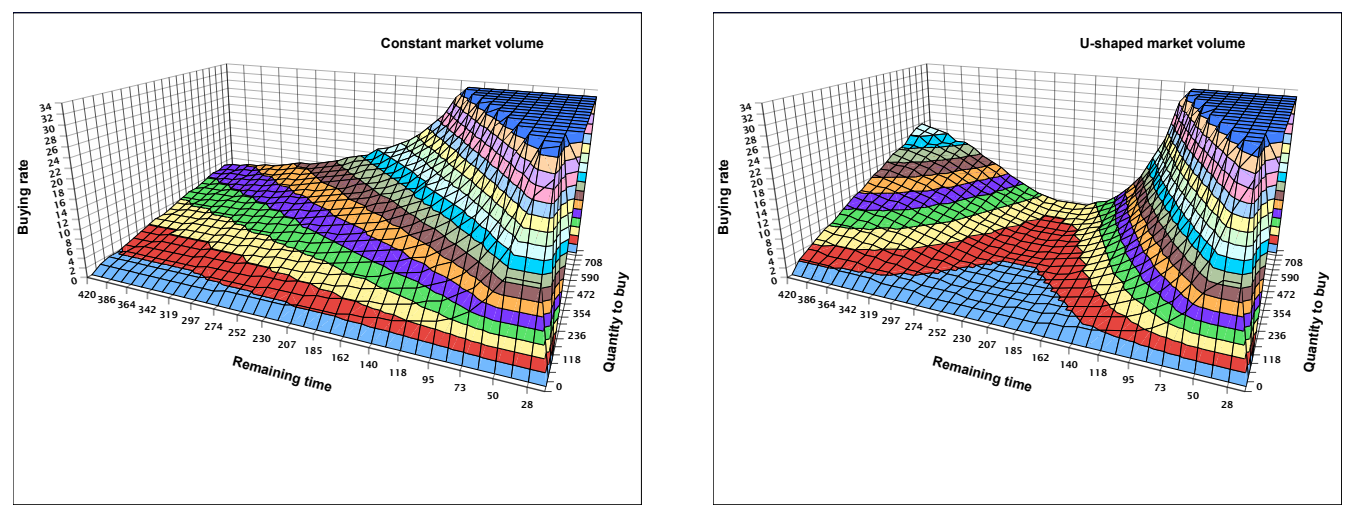

Figure 1: Optimal buying rate for different market regimes.

In Figure 1, we compare the case where the market volume is constant $V_{t}=100.000$, on the left, to the case where the market volume is strongly U-shaped: $V_{t}=100.000(1.1-\sin (\pi t / T))$. Both figures provide the optimal buying rate in terms of the remaining time $T-t$ and the remaining quantity to buy $Q_{t}$, for $S_{t}=S_{0}$. As expected the rate strongly decreases in the middle of the day, when the market volume is low and the impact on the price of the stock is high. This is compensated by a higher rate at the beginning of the day.

\subsection{Example 3}

We finally consider a similar case as the previous one except that the controller as an incentive to buy the shares more or less quickly. This can be modeled by adding a process $Z^{\nu}$ corresponding to the number of stocks sold during a trading period. Namely,

$$
Z_{t}^{\nu}:=\int_{\tau_{i}^{\nu}}^{t} \nu_{s} d s \text { for } t \in\left[\tau_{i}^{\nu}, \tau_{i}^{\nu}+\delta_{i}^{\nu}\right)
$$

By convention, we set $Z^{\nu}=Z_{\tau_{i}+\delta_{i}}^{\nu}$ on $\left[\tau_{i}^{\nu}+\delta_{i}^{\nu}, \tau_{i+1}^{\nu}\right)$. The aim of the controller is then to minimize

$$
\mathbb{E}\left[\ell\left(Y_{T}^{\nu}+\left(S_{T}+c\left(Q_{T}^{\nu}, S_{T}, V_{T}\right)\right)\left(Q_{T}^{\nu}\right)^{+}\right)+f\left(T,\left(Q_{T}^{\nu}\right)^{+}\right)+\sum_{i \geq 1} \mathbf{1}_{\tau_{i}^{\nu}+\delta_{i}^{\nu} \leq T} f\left(\tau_{i}^{\nu}+\delta_{i}^{\nu}, Z_{\tau_{i}+\delta_{i}}^{\nu}\right)\right]
$$

where the dependence of $f$ in time means that the controller prefers the buy quickly, i.e. $f$ is increasing in time, or take more time, i.e. $f$ is decreasing in time.

\section{References}

[1] R. Almgren. Optimal trading in a dynamic market. Technical Report 2, 2009. 
[2] R. Almgren and B. Harts. A dynamic algorithm for smart order routing. Technical report, StreamBase, 2008.

[3] R. Almgren and J. Lorenz. Bayesian adaptive trading with a daily cycle. Journal of Trading, 2006.

[4] R. F. Almgren and N. Chriss. Optimal execution of portfolio transactions. Journal of Risk, 3(2):5-39, 2000.

[5] G. Barles and P. E. Souganidis. Convergence of approximation schemes for fully nonlinear second order equations. Asymptotic analysis, 4:271-283, 1991.

[6] D. Bertsimas, A. W. Lo, and P. Hummel. Optimal control of execution costs for portfolios. Computing in Science and Engg., 1(6):40-53, 1999.

[7] B. Bouchard and N. Touzi. Weak dynamic programming principle for viscosity solutions. Technical report, CEREMADE, 2009.

[8] M. Crandall, H. Ishii, and P.-L. Lions. User's guide to viscosity solutions of second order partial differential equations. American Mathemtical Society, 27:1-67, 1992.

[9] K. J. Falconer. The Geometry of Fractal Sets (Cambridge Tracts in Mathematics). Cambridge University Press, July 1986.

[10] J. Gatheral. No-dynamic-arbitrage and market impact. Social Science Research Network Working Paper Series, October 2008.

[11] P. Hewlett. Optimal liquidation against a markovian limit order book. Quantitative Methods in Finance Conference, 2007.

[12] R. Kissell and R. Malamut. Understanding the profit and loss distribution of trading algorithms. Technical report, JP Morgan, February 2005.

[13] B. J. Lapeyre, A. Sulem, and D. Talay. Understanding Numerical Analysis for Option Pricing. Cambridge University Press, February 2009.

[14] C.-A. Lehalle. Rigorous strategic trading: Balanced portfolio and mean-reversion. The Journal of Trading, 4(3):40-46.

[15] C.-A. Lehalle. Rigorous optimisation of intra day trading. Wilmott Magazine, November 2008.

[16] G. Pagès, S. Laruelle, and C.-A. Lehalle. Optimal split of orders across liquidity pools: a stochatic algorithm approach. Technical report, 2009.

[17] M. Wyart, J.-P. Bouchaud, J. Kockelkoren, M. Potters, and M. Vettorazzo. Relation between bid-ask spread, impact and volatility in double auction markets. Technical report, March 2006.

[18] L. Zhang, P. A. Mykland, and Y. A. Sahalia. A tale of two time scales: Determining integrated volatility with noisy high-frequency data. Journal of the American Statistical Association, 100(472), 2005. 